\title{
La figure mythique du chamane dans ses représentations audiovisuelles occidentales
}

The mythical figure of the shaman in its audio-visual Western representations

\section{Charles Stépanoff}

\section{(2) OpenEdition}

1 Journals

\section{Édition électronique}

URL : https://journals.openedition.org/emscat/462

DOI : $10.4000 /$ emscat.462

ISSN : 2101-0013

\section{Éditeur}

Centre d'Etudes Mongoles \& Sibériennes / École Pratique des Hautes Études

\section{Édition imprimée}

Date de publication : 1 décembre 2004

Pagination : 17-68

ISBN : 2-9518888-2-1

ISSN : 0766-5075

Référence électronique

Charles Stépanoff, «La figure mythique du chamane dans ses représentations audiovisuelles occidentales », Études mongoles et sibériennes, centrasiatiques et tibétaines [En ligne], 35 | 2004, mis en ligne le 31 mai 2014, consulté le 13 juillet 2021. URL : http://journals.openedition.org/emscat/462 ; DOI : https://doi.org/10.4000/emscat.462

Ce document a été généré automatiquement le 13 juillet 2021.

(c) Tous droits réservés 


\title{
La figure mythique du chamane dans ses représentations audiovisuelles occidentales
}

The mythical figure of the shaman in its audio-visual Western representations

\author{
Charles Stépanoff
}

1 Les mythes de la société occidentale ne sont pas plus que ceux de n'importe quelle société des créations fantaisistes et gratuites, à repousser en raison de leur manque de conformité avec la réalité. Dans la mesure où il assume une fonction dans la société qui l'a produit, un mythe ne peut être considéré comme un simple «mensonge » ou une « erreur ». En Occident, le chamane est une figure mythique, c'est-à-dire une identité construite, qui apparaît dans plusieurs de nos productions culturelles. C'est la culture occidentale qui, pour le substituer à celui de sorcier, a créé le concept de chamane, concept suffisamment vague pour pouvoir s'appliquer à des individus appartenant aux sociétés les plus diverses et les plus éloignées, et suffisamment précis cependant pour qu'il impose sa marque reconnaissable et familière dans des œuvres multiples qui sont autant de variantes du mythe. Face à l'expansion rapide de ces variantes, il nous a paru utile de nous interroger sur le besoin propre à notre sensibilité contemporaine qui a rendu nécessaire la création récente d'une telle figure dans notre imaginaire.

2 Le corpus utilisé pour cette étude sera celui des productions audiovisuelles, principalement françaises, qu'elles soient télévisuelles ou cinématographiques, fictionnelles ou documentaires ${ }^{1}$. Le code des images et des sons permet de véhiculer des représentations qui ne trouvent plus d'expression dans les productions écrites en raison d'une censure implicite les déclarant périmées. C'est le cas par exemple des notions de «sauvage», de "primitif» ou de «peuple sans histoire». Mais la suppression du mot n'entraîne pas toujours l'oubli de l'idée, et la production audiovisuelle est devenue le refuge de mythes peu avouables, mais visiblement indispensables à la conscience occidentale.

3 Les films faisant intervenir la figure du chamane sont des œuvres destinées à un public occidental. Quand bien même certains réalisateurs scrupuleux, fidèles à la 
recommandation de Jean Rouch, tâcheraient de montrer le résultat de leur travail aux personnes filmées, le sens et les techniques mises en œuvre dans leur montage demeurent des produits de leur propre culture. Pourtant ces films ont tous un statut ambigu. Aucun d'entre eux, même les films de fiction, même les œuvres les plus personnelles, ne se veut la pure et simple expression de l'intériorité de l'auteur dans un médium propre à la tradition occidentale. S'il s'agit d'" œuvres d'art", elles sont particulières, car elles n'expriment pas seulement les affects de l'artiste, elles donnent une information sur le monde, elles se veulent un miroir renvoyant à une réalité extérieure au film, à l'artiste, et surtout à la société occidentale. Dissimulant son statut de témoin d'une rencontre entre sujets de deux cultures, le film se donne comme représentation transparente et pure d'un état de faits exotiques, où les filmés ne sont plus des hôtes mais des Autres, où la différence relative est objectivée dans une étrangeté de nature. La démarche de l'anthropologue s'en distingue dans la mesure où il reconnaît que sa position est engagée dans une relation, et que son travail, en définitive, ne s'évade pas des limites d'« une conversation de l'homme avec l'homme » (Lévi-Strauss [1973] 1997, p. 20).

4 Il est vrai que les principes qui guident la fabrication d'un film ne ressemblent guère à ceux d'une enquête anthropologique. Le réalisateur n'est pas un chercheur qui puisse mener à bien, seul, un travail indépendant. Il n'est pas le propriétaire unique de son travail, celui-ci répond souvent à une commande, et associe une équipe nombreuse. Le principe qui guide le fabricant de film n'est pas non plus, bien sûr, le mensonge, pas plus que le conteur de mythe ou d'épopée n'est un mythomane. Son principe est plutôt celui d'un arbitrage entre un modèle propre à sa culture, partagé par lui, le diffuseur et le spectateur, et, d'autre part, le chaos des situations et des informations auxquelles il est confronté sur le lieu du tournage. Comment le fabricant de film s'y prend-il pour mettre en ordre un matériau épars, quelles sont les techniques et les stratégies mises en œuvre pour transformer les données informes et paradoxales du terrain en une figure mythique reconnaissable par les spectateurs? Nous chercherons à dégager les lignes de forces, les traits fondamentaux du modèle, c'est-à-dire le visage du chamane mythique, tel qu'il transparaît au-delà des concessions faites aux situations locales et de la variété des productions. C'est par l'ensemble de ses versions qu'il faut définir le mythe, disait Lévi-Strauss.

5 Sans systématiquement passer au crible les informations données par les films, ce qui serait aussi stérile que de chercher à vérifier l'authenticité des événements d'une épopée, nous relèverons à l'occasion certains traits qui s'écartent avec le plus d'évidence des données de terrain, et qui se signalent donc comme des caractères propres au modèle occidental du chamane particulièrement importants pour le fabricant de film.

\section{Les sources}

6 Nous avons utilisé principalement pour cette recherche les ressources de l'INAthèque. Dans le catalogue des « archives de l'INA » (antérieur à 1992), le mot « chamane » n'est pas utilisé comme "descripteur» (mot-clef). Il est seulement un sous-descripteur inclus dans le domaine du descripteur « sorcier ». À partir de la création du dépôt légal en juin 1992, un descripteur spécifique est créé dans le nouveau catalogue (et même deux en raison de la double orthographe « chamane » et «chaman »). Les productions audiovisuelles faisant intervenir la figure du chamane sont principalement des films 
documentaires, souvent commandés par les chaînes télévisées, mais aussi des fictions destinées au cinéma, et également des émissions pédagogiques. Les désignations de genre sont celles données par les documentalistes de l'INA. Nous citons quelquefois le « résumé producteur » tiré du catalogue, c'est-à-dire une présentation du film par la société de production, et dont le texte reprend des extraits du commentaire en les synthétisant.

\section{La fabrication du chamane}

7 Le mot chamane, d'origine toungouse, figure rarement dans les terminologies employées par les individus que le fabricant de films désigne sous ce terme ${ }^{2}$. C'est à l'Occidental que revient la responsabilité de reconnaître ou non le chamane. On le voit bien dans l'explication suivante des réalisateurs du film Indo Pino tourné en Indonésie: «C'est ainsi que les Wana nomment leurs chamans : les Taw-Waliya, "les gens de la forêt". " ${ }^{3}$ L'Occidental voit un chamane, avec autant d'évidence qu'il reconnaît un lion ou un figuier, et ensuite seulement il s'enquiert de la dénomination locale pour nous la fournir.

Or le « chamane » est d'abord une figure créée par l'Occident. Si le mot lui-même vient des Toungouses, la signification et la connotation qu'il a pour nous ne sont plus les mêmes que celles qu'il pouvait avoir pour les locuteurs de ce peuple. Le Petit Robert donne du mot la définition suivante: "Prêtre-sorcier, à la fois devin et thérapeute, dans les civilisations d'Asie centrale et septentrionale et par ext. dans d'autres civilisations. " Si l'on peut, à la rigueur, faire l'hypothèse que les Toungouses tomberaient d'accord avec le début de la définition, en revanche la deuxième partie, qui donne au mot une extension géographique, est un ajout spécifiquement occidental. Le chamane est un homme qui appartient à une autre civilisation, une civilisation lointaine. Il est le prêtre ou le sorcier non pas des nôtres, mais des autres. Nourrie par les observations et les inventions des voyageurs, la figure du chamane s'est constituée pour former aujourd'hui un personnage dont les traits quelquefois contradictoires n'en sont pas moins caractéristiques.

9 La fabrication d'un personnage mythique ne pose guère de difficultés pour les films de fiction, qui, par définition, doivent rendre visibles des faits et des êtres imaginés par le scénariste. L'opération semble moins évidente pour les films documentaires. La réalité, suppose-t-on, indique d'elle-même qui est le chamane, et quel il est. En fait, face aux données du terrain, c'est le fabricant de film qui décide si un individu est un chamane ou non. Il peut nommer « chamane » une personne qui ne connaît pas ce mot (Les deux fils du chaman, Indo Pino, etc.), mais aussi une personne qui connaît le mot et ne se l'applique pas. Ainsi, dans un reportage de Canal Plus intitulé Spiritualité: chamane à Moscou, un musicien rockeur inspiré par le chamanisme et pratiquant le chant diphonique était nommé "chamane " dans le commentaire en voix off et dans la présentation du producteur, alors que lui-même dans son interview ne s'applique jamais à soi ce titre et conseille modestement de s'adresser à de vieux chamanes sibériens. Mais le fabricant de film est le mieux placé pour connaître la représentation occidentale du chamane, et c'est lui qui détermine si une personne est capable de l'incarner ou non.

Le chamane doit transmettre un message à l'Occident. Pourtant il est dans les caractéristiques de la figure du chamane d'être muette, aussi le fabricant de film doit-il 
souvent faire appel à des intervenants tiers pour exprimer le discours chamanique, par exemple à un ethnologue local (Taïga, terre des chamans). Mais le plus souvent, c'est le commentateur occidental qui transmet le message chamanique par l'usage abondant du discours indirect libre, procédé cher à Flaubert qui permet d'établir un flou empathique sur l'origine de la parole. Le commentateur du documentaire Le voyage de Charlie explique ainsi à propos d'un chamane shipibo: "Guillermo est peu à peu entré en contact avec le monde des esprits. » Est-ce la conviction intime du commentateur admiratif ou celle du chamane, qui n'est cependant pas interrogé à ce sujet ? Lequel des deux croit à l'existence d'un deuxième monde, le « monde des esprits »? Probablement ni l'un ni l'autre: cette phrase n'a d'autre but que d'exprimer la représentation occidentale des représentations indigènes.

11 Il faut encore signaler l'importance décisive du doublage dans le remodelage des données du terrain. La précision des traductions n'est pas une obligation, et le ton souvent dramatique donné par le comédien récitant permet d'orienter dans le sens désiré la signification des paroles si leur contenu n'est pas assez expressif et touchant (Taïga, terre des chamans, Les Aïnus de Hokkaido).

12 Un cas extrême de procédé de fabrication dès la prise de vue est illustré par le documentaire intitulé Les nomades $d u$ Grand Khan. Dans ce film atypique, la figure du chamane est incarnée par une comédienne visiblement habituée à jouer ce rôle sur la scène des théâtres. Les fabricants du film ont dû réaliser un arbitrage entre les signes de l'authenticité, propres au genre documentaire, et les traits caractéristiques du mythe occidental du chamane. Sans doute confrontés à des chamanes mongols entrant difficilement dans les normes des représentations occidentales, ils ont préféré sacrifier les signaux du "pris sur le vif » au prestige de l'illustration conforme d'une figure mythique. La chamane est donc ici une jeune femme fardée aux gestes lents et théâtraux, portant un costume de théâtre sorti pour la première fois dans la nature ; mais du moins sa «transe " a bien ce quelque chose d'« extatique " qui manque si souvent aux chamanes rencontrés sur le terrain. Le documentaliste de l'INA a su, dans la notice du film, consigner ces deux aspects, l'inauthenticité et la conformité au mythe : «Ce document met en scène une femme chamane. [...] Pour la caméra, elle accomplit un rite ayant pour but d'entrer en contact avec l'esprit des morts. » C'est bien la figure du chamane que le documentaliste reconnaît, et son contact traditionnel avec les esprits grâce à la transe, - mais ce qu'il voit aussi, c'est la "mise en scène " "pour la caméra", observations sévères qui jettent un discrédit sur la nature documentaire du film.

13 S'il est extrême, ce cas ne l'est pas parce qu'il "met en scène » ce qui devrait être « spontané et naturel ». De nombreux autres documentaires, soutenus par des autorités savantes prestigieuses comme le C.N.R.S., ont recours à une part incompressible de mise en scène. Celle-ci est souvent révélée par la position de la caméra installée avant que l'un des protagonistes n'entre dans le champ tandis que l'autre l'attend (Les deux fils du chaman). Dans les tournages de documentaires télévisuels commerciaux, la pratique des répétitions permettant plusieurs prises est du reste courante, parfois considérée comme indispensable, pour les scènes non dialoguées, et même certaines scènes dialoguées brèves. Là où le film Les nomades $d u$ Grand Khan est exceptionnel, c'est par l'utilisation d'un acteur-chamane, c'est-à-dire d'une personne dont la profession même est de représenter la figure mythique du chamane sur les scènes des théâtres. La tâche du fabricant de film est ainsi considérablement simplifiée. Mais s'il n'est pas accompli à 
la prise de vue, le travail de mise en conformité doit de toute façon être exécuté : cette étape a lieu d'ordinaire à la post-production, par le dérushage, le montage et l'étalonnage, comme nous le verrons dans quelques exemples. Le dérushage permet de trier le matériel qui répond aux attentes du fabricant de film: cette opération a une importance considérable quand on sait la proportion des éléments filmés rejetés (de 90 à $99 \%$ ). À chaque moment de la fabrication du film, depuis la délimitation du champ par le cadrage et du temps par la durée des prises, jusqu'à la recréation des couleurs au moment de l'étalonnage, ultime étape de la fabrication, des choix sont opérés en fonction de critères précis. Tout au long de ce travail collectif nous voyons se modeler, sous les doigts experts des scénaristes, des réalisateurs, des éclairagistes, des cadreurs, des preneurs de son, des monteurs, des mixeurs, et des étalonneurs, la figure classique du chamane, inflexible et sereine.

\title{
Les fabricants
}

Comme le film est une production collective, nous désignons sous le terme indifférencié de « fabricant de film » tous ceux qui sont associés à l'écriture, au tournage et à la postproduction du film. En France, le personnage principal parmi les fabricants de film est le réalisateur. Aucun des réalisateurs dont nous avons consulté les œuvres n'est spécialisé dans le chamanisme. Seul Mark Soosaar est l'auteur de deux films faisant intervenir des chamanes. Le métier ne permet pas de spécialisation, car les réalisateurs sont amenés, suivant les commandes, à s'intéresser aux thèmes les plus divers (le pain, le disque, la mer Méditerranée, le rôle du père, l'homo sapiens, etc.) Les réalisateurs ne s'intéressent à leur sujet que pendant une période de quelques mois et n'ont pas le temps d'acquérir de renseignements très détaillés. Ils gardent donc une relative pureté au moment de reconstruire la figure mythique du chamane, et les influences qu'ils subissent des situations empiriques restent toujours limitées. Le système français de production des films documentaires ne permet qu'une prise de connaissance tardive de ces dernières. Les chamanes vivent dans des pays lointains et le financement du repérage, qui est quelquefois la première prise de contact du réalisateur avec son sujet, ne peut se faire qu'après l'adoption du projet par la société de production et le début d'un financement de ce projet par le Centre National de la Cinématographie. Or le C.N.C. ne finance pas un projet pour lequel une société de diffusion n'a pas donné son accord, ce qui signifie que le producteur doit avoir convaincu un diffuseur par un dossier complet avec un scénario précis, - sauf si le réalisateur est une autorité, c'est-àdire s'il a réalisé de nombreux films, ce qui implique nécessairement une absence de spécialisation.

\section{L'« Autre » par nature}

\author{
C'est une autre humanité que nous observons, \\ une autre conception de la vie \\ (Message d'un monde perdu, commentaire)
}

\section{L'inaccessible}

15 Tout d'abord le chamane a pour caractéristique dans ses représentations occidentales d'être difficile à atteindre. Il appartient à un petit peuple ou une « tribu » isolée dans la 
nature, sans voies de communication, avec peu de contacts extérieurs. L'héroïne éponyme du film documentaire Indo Pino est une chamane indonésienne de la tribu des Wana qui vivent «au cœur d'une forêt hostile». Les réalisateurs insistent sur l'isolement de cette société : «Il est essentiel, pour comprendre [les représentations des chamanes sur la santé], de signaler que, jusqu'ici en tout cas, les Wana n'ont jamais eu accès aux thérapies médicales modernes venant d'Occident", et, "à part quelques ethnologues et quelques chercheurs, nous sommes parmi les seuls Occidentaux à avoir des contacts aussi étroits avec eux $»^{4}$.

Dans Message d'un monde perdu, le commentaire souligne les difficultés extrêmes rencontrées par les équipes Cousteau pour progresser à la rencontre des chamanes dans « les régions les plus secrètes d'Amazonie ». Un équipement très performant est nécessaire, détaillé par une collection de plans panoramiques évocateurs : voitures $4 \times 4$, hélicoptères, bateaux à moteurs et hydroglisseurs. Il ne faut rien de moins pour pénétrer la jungle, où, « perdus dans son immensité, des mondes cachés subsistent ».

Le film Les Tsaatan, ceux qui chevauchent les rennes suit l'itinéraire d'un éleveur de renne, fils de chamane, qui rend un culte à l'esprit de son père. Les premières phrases du commentaire sont les suivantes : «Cet homme s'appelle Bat, il vit là où personne ne vient jamais. Bat fait partie de la tribu des Tsaatanes. » Que les peuples et les tribus "isolés " entretiennent vraisemblablement des relations intenses avec leurs voisins importe peu. Il suffit qu'ils n'aient pas de relations directes avec l'Occident pour être déclarés en état d'isolement.

L'équipe de tournage doit, si possible, être la première à rencontrer le chamane et sa tribu. C'est le cas de l'équipe Cousteau : la cérémonie qu'elle présente est «filmée pour la première fois ». Mais ce n'est pas une obligation absolue, sinon la tribu filmée ne recevrait plus jamais de visite d'équipes de tournage : or, le tournage du film sur les Tsaatanes qui eut lieu en 1995 fut immédiatement suivi de deux autres tournages français en 1996 et 1997.

Nécessairement les peuples les plus isolés deviennent bientôt les plus visités. On le voit bien avec les emblématiques Yanomamis auxquels plusieurs films ont été consacrés dans le cadre de l'exposition Yanomami, l'esprit de la forêt à la Fondation Cartier de 2003. Entre 1984 et 1993, ils ont fait l'objet de 21 films et reportages français. L'isolement n'est pas intéressant en soi, il est valable dans la mesure où il est visible, c'est-à-dire que l'on doit pouvoir constater l'absence de contamination de la culture occidentale sur les corps indigènes. C'est bien le cas des Yanomamis, qui ne sont nullement isolés, soumis au contraire au contact permanent et menaçant des chercheurs d'or, mais qui, évoluant à moitié nus dans une forêt luxuriante, gardent cependant leur succès entier auprès des producteurs.

\section{L'homme-nature}

\section{Un langage non humain}

Homme-nature, le chamane reste à l'abri des effets de la culture (occidentale), au point quelquefois de ne pas maîtriser le langage. Dans ses variantes les plus pures, la figure du chamane est en effet une figure muette. Le chamane Savili de Taïga, terre des chamans apparait à la toute fin du film : sans qu'on sache ce qu'il y faisait, il surgit de la forêt profonde sur un traîneau de rennes, et rejoint sa famille. C'est alors sa femme qui 
monopolise la parole dans le montage ; lui prononce à peine quelques monosyllabes. La séquence de la séance débute immédiatement après et le chamane ne prononcera de tout le film qu'une phrase intelligible, adressée à la caméra à la fin du rite : «Vous n'avez rien vu, n'est-ce pas? », remarque absurde pour le spectateur qui vient de tout voir, témoignage presque attendrissant de sa naïveté et son incompréhension de la technique. Il arrive cependant que le spectateur voie le chamane parler dans les documentaires. Naturellement Savili n'était pas muet, et il parle certainement dans les rushes, mais son langage ne peut atteindre le statut de parole pour le spectateur que s'il est traduit par des sous-titres. Non sous-titrés, les sons qu'il produit demeurent un langage mystérieux, inaccessible à l'intelligence et à la communication, un langage surou infra-humain. C'est l'effet que produisent les chants en évenk (toungouse) que Savili prononce pendant la séance finale et les paroles qu'il échange avec l'assistance. Le commentaire d'Oulan-Bator, recouvrant le chant mongol d'une chamane accomplissant une séance, donne l'explication suivante: «Elle chevauche un langage connu d'elle seule, comme le font les enfants quand ils jouent à penser le monde avec leurs premiers mots. » Ainsi est justifiée l'absence de traduction. Puisque le chamane est en transe, son comportement et ses paroles n'ont rien de culturel, ils ne sont plus soumis à aucune contrainte sociale, le chamane est tout entier pour lui et non plus pour les autres ; son langage est la réalisation parfaite d'un mythe dont Wittgenstein n'a cessé de dénoncer la fascination et le caractère contradictoire, celui du langage privé ${ }^{5}$.

\section{Une vie en osmose avec la nature}

21 S'il a abandonné le langage des hommes, le chamane communique en revanche aisément avec les animaux, ce qu'illustre la séquence de Chamane de Bartabas dans laquelle Anatoli charme un troupeau de chevaux sauvages du son de sa guimbarde. La communication entre le cheval et le chamane est suggérée visuellement grâce à un champ-contrechamp de gros plans à faible profondeur de champ ${ }^{6}$ dont la valeur psychologique est connue. D'après $L a$ forêt, le chamane peut aussi communiquer avec les végétaux: «Le chamane est celui qui parle avec les arbres, c'est un poète, un artiste qui tend à être l'homme total» (résumé producteur). D'une manière générale, le chamane est un homme qui vit «en harmonie avec la nature». Ce point essentiel est répété inlassablement par les commentaires de la plupart des films. Les Évenks de Taïga, terre des chamans vivent "selon des croyances chamaniques millénaires dans l'harmonie avec le monde des morts et les forces du cosmos». La chamane du documentaire Les Aïnus de Hokkaido, le jardin où s'amusent les dieux partage cette idée : «Elle veut respecter la promesse de son peuple de vivre en harmonie et dans le respect de la nature."

La fusion perpétuelle du chamane avec son environnement doit être soutenue par des signaux visuels. Un plan spectaculaire l'illustre bien dans Spiritualité: chamane à Moscou. Pris en contre-plongée et en grand-angle, le plan montre le chamane en train de chanter perché sur la branche d'un arbre. Entouré par les seules frondaisons des bouleaux, semblable à un oiseau chanteur, il est un élément du royaume des plantes et des animaux. Le chamane possède une connaissance intime de la faune et surtout de la flore. La séquence classique de la recherche des plantes médicinales ou psychotropes se retrouve notamment dans Le voyage de Charlie (avec le chamane Guillermo), Les deux fils du chamane, Les enfants de la lune, Les Aïnus de Hokkaido ("En tant que chamane, Asiri a hérité du savoir des anciens sur la connaissance des arbres et des plantes 
médicinales ») et Message d'un monde perdu. Grâce aux fruits de sa cueillette, le chamane peut ainsi « vivre autant que possible en autarcie, de la manière la plus proche de celle de ses ancêtres, en n'ayant recours qu'à des produits naturels ou bio-dégradables » (Les Aïnus de Hokkaido).

\section{Le philosophe du dualisme et de la pluralité des mondes}

Le chamane a conscience de l'existence d'un monde parallèle à celui qu'expérimentent les sens, le «monde des esprits». Cette dernière expression est la plus courante, mais on rencontre d'autres désignations comme "l'autre monde" (Message d'un monde perdu), «le monde des ténèbres» (ibid.), "le monde des morts» (Taïga, terre des chamans), « le monde de l'au-delà » (Les chamanes de la préhistoire). Ce monde correspond à ce que l'un des principaux artisans de la représentation occidentale du chamane, Michael Harner, appelle la "réalité non ordinaire " qui ne se laisse percevoir qu'en "état de conscience chamanique " (Harner 1982, p.16). Il est caractérisé par son étrangeté et son aspect effrayant. S'il n'est presque jamais illustré dans les films au niveau visuel en raison des difficultés techniques qu'on devine, le monde des esprits signale souvent sa présence dans la bande sonore. Un son particulièrement prisé pour le chamanisme sibérien est celui de la guimbarde, systématique dans Chamane de Bartabas, épisodique dans Molom. Le chamane, en rapport avec ce monde étrange, est par cette position un être extraordinaire. Le seul nom du chamane, lorsqu'il est prononcé pour la première fois, est souvent accompagné d'effets puissants destinés à attirer l'attention du spectateur sur le caractère exceptionnel et mystérieux du personnage. Dans le film de Bartabas, Anatoli au moment de mourir prononce la phrase suivante : «Je sais ce que je dis. Je suis chamane... Je connais la puissance des esprits.» À l'image, on voit un insert ${ }^{7}$ sur les yeux de l'acteur. Au moment où est prononcé le mot «chamane", ses yeux s'élargissent puissamment. Puis se fait entendre une musique aiguë, très lente, aux accents mystérieux.

Quant à représenter visuellement les visions chamaniques, un seul film s'y est risqué : c'est le récent Blueberry de Jan Kounen. Des images de synthèse baroques mêlant plantes, serpents et cercles concentriques ambitionnent de plonger le spectateur dans le monde des esprits. La critique a sanctionné avec sévérité cette tentative («bestioles en images de synthèse » selon l'A.F.P. du 9 février 2004, "délires graphiques spectaculaires qui évoquent comme un fond d'écran surchargé de motifs » selon Le Monde du 11 février 2004) en raison de son évident échec esthétique, mais aussi, plus profondément, parce qu'une telle expérience réduisait ouvertement l'altérité inaccessible dans laquelle doit rester enfermée la pensée chamanique. Le scandale ne vient pas tant de ce que le réalisateur donne des croyances indiennes une illustration arbitraire que ne vient soutenir aucun protocole d'enquête, il vient plutôt du fait même de donner une image à ce qui ne vaut pour nous, Occidentaux, que par son obscurité.

L'exposition Yanomami, l'esprit de la forêt présentée en 2003 par la Fondation Cartier pour l'art contemporain (« l'exposition la plus surprenante de l'année » selon le journal Libération ${ }^{8}$ ) a également tenté, mais avec plus de succès, de rompre avec la tradition d'invisibilité du monde des esprits. Parmi de nombreux autres artistes, cinéastes et photographes, le plasticien français Vincent Beaurin présentait des sculptures en polystyrène jaune couvertes de paillettes. Dans un reportage diffusé au journal télévisé d'Arte $^{9}$, le commentaire suivant accompagnait les images des sculptures filmées en 
gros plans: "Quand les chamanes voient les esprits des ancêtres animaux, ils sont scintillants et magnifiques. L'œuvre est du Français Vincent Beaurin qui n'a pas été en Amazonie. » L'idée est de représenter matériellement les représentations yanomamies, sans qu'il soit besoin pour cela de prendre connaissance de leurs éventuelles manifestations pratiques et symboliques chez les acteurs. Pour l'artiste, la connaissance des pensées des Yanomamis est directe et sans intermédiaire. C'est également l'explication que donne le journal Libération: «[...] les images mentales des chamans yanomamis venant inspirer les projets d'artistes » (op. cit.). Le parallèle entre chamane et artiste n'est évidemment pas anodin : l'artiste est l'alter ego du chamane dans nos sociétés. L’idée était déjà présente dans La forêt (« Le chamane est celui qui parle avec les arbres, c'est un poète, un artiste qui tend à être l'homme total $\left.{ }^{10}\right)$. Le reportage d'Arte continuait avec une interview de Davi Kopenawa, «chamane yanomami » invité à Paris : «Quand je vois ces choses-là, ça m'étonne, ça me surprend que les Blancs aient une certaine connaissance de ces choses. Ils ne sont jamais venus dans la forêt, ils ne sont jamais venus chez nous, mais quand même ils ont des aperçus de ce que voient les chamanes. » Le chamane était donc simplement invité à donner sa confirmation, son blanc-seing aux œuvres des artistes français. Typiquement, dans cette exposition qui affirmait « confronter les images des artistes aux visions des chamanes » (commentaire Arte), aucune production indigène n'était présentée pour faire face aux œuvres d'art des Occidentaux. En réalité, l'exposition ne confrontait pas deux mondes, mais présentait diverses illustrations photographiques, filmiques et plastiques de la figure mythique du chamane, ou plus largement du sauvage, dans l'imaginaire occidental.

\section{Le « spécialiste de la transe »}

« Le chamane est le spécialiste d'une transe pendant laquelle son âme est censée quitter le

corps pour entreprendre des ascensions célestes ou des descentes infernales", nous dit Mircea Eliade, historien des religions

(Le chamanisme aujourd'hui, « Archimède ", commentaire).

La phrase d'Eliade, citée en exergue au début de l'émission pédagogique "Archimède ", résume la doxa qui est partagée et défendue par la grande majorité des productions audiovisuelles consultées. Le chamane est un homme caractérisé par la maitrise d'une expérience intérieure particulière. Cette expérience est nommée "transe ", " extase " ou encore "état de conscience modifié » ${ }^{11}$. C'est elle qui permet au chamane de pénétrer dans le fameux «monde des esprits ». Suivant cette explication psychologique intériorisante, l'acte chamanique n'est pas un acte social, mais une expérience à la fois mystique et sensorielle d'ordre privé.

L'émission pédagogique Animisme et chamanisme, du magazine "Des religions et des hommes ", fait intervenir une autorité du monde scientifique, Jean Delumeau, membre de l'Institut, professeur honoraire au Collège de France. Il définit le chamane comme « un prêtre magicien pratiquant la transe, la divination et des guérisons ». Ces paroles sont suivies de sons de trompe graves et profonds, chargés de faire ressentir le mystère des phénomènes évoqués. 
28 La " séance ", au cours de laquelle le chamane entre en transe, est, par conséquent, un élément incontournable et essentiel du film à chamane. Stéphane Bégoin, le réalisateur du documentaire Le voyage de Charlie, en témoigne par son insistance : "J'étais allé en repérage en Amazonie pour rencontrer Guillermo [chamane shipibo] et assister à des séances, pour être bien sûr que lui aussi accepterait de se joindre à notre projet. C'était une condition indispensable parce que sans les séances filmées je ne voulais pas faire le film. ${ }^{12}$ Souvent plusieurs effets d'annonce viennent éveiller l'attention du spectateur et le mettre dans l'attente de l'événement qui se prépare. Taïga, terre des chamans est particulièrement riche en effets de ce type. Au milieu de plusieurs séquences, dès le début du film, des inserts extraits de la séance sont calés entre les plans sans rapport. Ce sont des pendeloques du costume du chamane qu'on verra apparaître en entier seulement à la fin du film. Leur agitation permet de comprendre que le propriétaire est en train d'accomplir une séance, et un ralenti ajoute à leur étrangeté.

On rencontre également des effets d'annonce sonore : ce sont par exemple des éléments de la piste de son accompagnant la transe ajoutés au son in ${ }^{13}$ de la scène à l'image (Chamane), le bruit d'un vent violent (Les nomades du Grand Khan), ou le son d'une guimbarde. Enfin, on peut tout simplement avoir recours au commentaire pour signaler au spectateur l'importance de ce qu'il va voir. Dans Oulan-Bator, quand Mme Bayar se prépare, le commentateur annonce sur un ton grave: "Elle va jouer une pièce aussi vieille que le monde. »

Puisque la transe a sa valeur en elle-même, puisqu'elle est une expérience intime et non un acte rituel doué d'une signification collective, le fabricant de film documentaire n'hésitera pas à demander au chamane de faire une séance sur commande. C'est ainsi qu'a agi Mark Soosaar faisant jouer pour la caméra un chamane khante dans Le père, le fils et le saint Torum ${ }^{14}$, et c'est aussi le cas, comme nous l'avons vu, dans le film Les nomades du Grand Khan.

31 La transe se manifeste extérieurement de manières diverses : par un état d'agitation, ou au contraire par une léthargie profonde (on devrait parler dans ce cas d'« extase »). Dans tous les cas, les signes d'un état anormal se manifestent. Mis en valeur par un cadrage serré, les yeux du chamane jouent un rôle important; ils peuvent être exorbités (Le voyage de Charlie, Les nomades du Grand Khan), ou au contraire fermés (Molom, Chamane de Bartabas, Les Aïnus de Hokkaido).

La bande-son joue un rôle essentiel dans la scène de la transe. Pour les documentaires, le son synchrone est souvent abandonné : c'est le signal d'un moment particulièrement important, où rien ne peut être laissé au hasard, et où les données du terrain doivent naturellement laisser la place à une reconstitution professionnelle et conforme de la figure mythique du chamane. Le son est chargé d'évoquer le mystère, le caractère non ordinaire du phénomène, ce à quoi le son direct, naturellement prosaïque, ne saurait parvenir, bien au contraire. Dans Le voyage de Charlie, il est totalement éliminé pour les séances, qui sont au nombre de trois. Dans la première et la deuxième, c'est de la musique d'orgue qui se fait entendre, à laquelle s'ajoutent divers enregistrements de chuchotements également postsynchronisés ${ }^{15}$ et superposés. Une autre piste est consacrée à des chants traditionnels affectés d'un effet de résonance. Une fois l'ambiance installée, une piste supplémentaire entre en action, celle du commentaire en voix off. Rien ne permet mieux de créer une atmosphère à la fois d'étrangeté, d'intimité et d'espace invisible que la musique d'orgue affectée d'un écho - le filtre numérique à effet d'écho étant d'ailleurs d'un usage extrêmement fréquent pour les 
sons de séance postsynchronisés. Tout est fait pour évoquer l'ambiance d'une cathédrale, qui visiblement demeure plus évocatrice du phénomène mystique que celle d'une maison de pisé dans la forêt péruvienne. La pratique n'est pas nouvelle, Roland Barthes la relevait à propos du film Continent perdu où « toutes les scènes "bouddhistes" sont soutenues par un vague sirop musical, qui tient à la fois de la romance américaine et du chant grégorien » ([1957] 1970, p. 153).

Le travail de l'image n'est pas moins complexe. À la première séance du film de Stéphane Bégoin, l'Inuit Charlie est invité à fumer une pipe à tabac dont la fumée transporte les émanations d'une substance psychotrope placée à proximité ${ }^{16}$. Le chamane participe bien sûr aux inhalations, ainsi que quelques autres personnes. Tous sont assis par terre le dos aux murs de la maison. Comme on l'apprendra plus tard, l'effet de cette séance aura été assez décevant pour Charlie qui n'est pas parvenu à se concentrer ; les moyens mis en œuvre à la post-production resteront donc sobres. Le début de la séance a été filmé avec une balance des blancs ${ }^{17}$ très bleutée, destinée traditionnellement à donner un effet de mystère et d'étrangeté. Les plans sont presque entièrement monochromes sauf autour des bougies nimbées de rouge. Puis les bougies sont soufflées, et l'on passe à un noir complet. Ce noir artificiel est remplacé par des plans moyens pris en caméra infrarouge, dont l'effet est de donner une monochromie absolue calée sur un bleu grisé à l'étalonnage, et surtout de produire des effets de solarisation. Le résultat le plus impressionnant est sans doute celui des yeux qui apparaissent dans ce mode de prise de vue comme de petites billes lumineuses. L'étrangeté de la scène atteint un degré extrême.

34 La troisième séance est "réussie » d'après le témoignage ultérieur de Charlie : il a pu communiquer avec les esprits de ses ancêtres. La post-production se doit donc d'assurer des effets encore plus saisissants que ceux des deux premières. La séance est introduite par un fondu-enchaîné exceptionnellement long superposant trois plans. Le premier plan est le dernier d'une séquence de paysages : il s'agit d'un travelling avant au milieu d'une rivière en direction d'un magnifique soleil couchant, la caméra étant posée sur un bateau : un plan splendide. Le deuxième est un plan tourné en vitesse lente dans la salle où se passe la séance. La vitesse lente (de 3 à 10 images par seconde) permet de déréaliser les mouvements qui, saccadés, dessinent des traces sur le cadre. La balance des blancs est très bleue, la même que pour les premières séances. Enfin un troisième plan intervient au même moment que le deuxième mais pas immédiatement discernable: il s'agit d'un insert sur des volutes de fumée en fond transparent. Les volutes blanches se déplacent au milieu de la séance. Filmées en vitesse normale, elles font ressortir l'étrangeté de la scène en arrière-plan filmée en vitesse lente. À la fin de la séance, on entend en off le commentaire ultérieur de Charlie sur son expérience : il a vu un nuage, ce que la post-production s'est chargée de nous montrer.

L'effet total de cette séquence contraste avec les propos du réalisateur, pour qui l'utilisation de la caméra infrarouge était un progrès dans le sens du réalisme puisqu'elle permettait de supprimer l'éclairage d'appoint qui aurait altéré l'ambiance. D’après le souvenir du réalisateur lui-même, l'ambiance de la séance était assez différente de celle qui est reconstituée dans le film:

Quand la séance commençait, il y avait juste Guillermo, deux personnes et Charlie. Guillermo s'occupait déjà de Charlie. C'est très long, on ne le voit pas dans le film. Entre le moment où ils commencent à prendre la boisson et celui où il commence à se passer quelque chose il y a au moins deux heures. Il faut que la boisson fasse son effet, ce n'est pas brutal, c'est très progressif. Puis à un moment donné, on ne savait 
pas trop pourquoi, d'autres Shipibo commençaient à arriver. C'était vraiment très curieux, il y en avait un, puis deux, puis trois, une femme avec son enfant, et ça durait très longtemps. Les séances durent en tout cinq à six heures ${ }^{18}$. elle aurait pu être signalée par le commentaire. Au contraire, le montage serré de plans brefs donne une impression de cadence rapide. Un élément remarquable qui, d'après l'interview, a frappé l'attention du réalisateur, c'est l'entrée de personnes venues de l'extérieur. Ce fait intéressant n'est pas montré dans le film, pour plusieurs raisons. En lui-même, il n'apporte rien à la représentation standard de la séance. Mais de plus il la gêne, car montrer l'entrée de personnes dans la pièce impliquerait l'apparition d'une lumière extérieure, des mouvements prosaïques d'installation, etc., qui rompraient l'ambiance «magique » de la scène. On voit ici typiquement le modèle occidental du sacré et de la cérémonie sacrée se confronter à des comportements indigènes contradictoires: c'est le travail de la production et de la post-production que de transformer les données empiriques en une réalité conforme au mythe traditionnel. privée. Tous les efforts du cadreur tendront donc à isoler le chamane de l'assistance qui l'entoure. Simon Xavier qui a cadré lui-même les images de son film Oulan-Bator, après quelques plans sur les spectateurs, fait se succéder des plans spectaculaires en contreplongée et même en plongée sur la chamane Bayar qui fait une séance dans un appartement. Elle apparaît ainsi comme un personnage extraordinaire, peu à peu isolé dans son monde parallèle à mesure que la transe s'installe. Dans Les deux fils $d u$ chamane, Medsinu, le fils du chamane mort, est à la fin du film investi comme successeur de son père et une cérémonie collective rassemble la communauté de la vallée. Des danses rituelles se prolongent toute la nuit tendant à faire entrer les danseurs en «transe ». Medsinu danse parmi les autres au milieu d'un public nombreux qu'il rejoint de temps en temps. Mais les techniques du film sont utilisées systématiquement pour isoler le chamane des autres membres de sa communauté. Un violent éclairage d'appoint, blanc, dont le faisceau étroit suit les mouvements de Medsinu, fait de lui le centre unique de l'action. De plus, des gros plans centrés sur son visage viennent mettre en évidence l'altération de ses traits. L'éclairage fait ressortir les gouttes de sueur qui coulent de son front. À aucun moment un plan d'ensemble ne vient présenter la situation générale et replacer la «transe " de Medsinu dans son contexte. Avec les gros plans psychologiques sur le visage de Medsinu alternent des plans plus larges (plans américains) sur certains membres du public. Cette alternance répétée crée un effet de champ-contrechamp, où le chamane, spécialiste de la transe, est le seul sujet de l'action, face à un public passif de spectateurs.

\section{Le passé en personne}

Le chamane est l'image même du passé incarné dans le présent. Il appartient souvent à un peuple extrêmement vieux. Les peuples où le chamane apparaît ont pour le moins «plusieurs milliers d'années d'existence » (les Tsaatanes, les Évenks, les Jivaros, etc.). Ainsi les Évenks (Taïga, terre des chamans) sont-ils les « ancêtres des Indiens d'Amérique du Nord ». On voit l'état d'extrême vieillesse d'ancêtres aussi reculés dans le passé : les Évenks sont en quelque sorte un peuple de spectres vivants.

Le chamane lui-même est ce qu'il y a de plus ancien dans son groupe. Les chamanes wana indonésiens du film Indo Pino se livrent à des «pratiques ancestrales», et

Études mongoles et sibériennes, centrasiatiques et tibétaines, 35 | 2004 
partagent des "représentations sans doute millénaires " ${ }^{19}$. Dans Oulan-Bator, les représentations chamaniques sont dites exister "depuis l'âge de pierre" (commentaire), et l'on apprend à propos de Mme Bayar qu'« elle exerce le plus vieux métier de Mongolie : elle est chamane ». Les chamanes de l'île de Cheju en Corée ne sont pas en reste, qui pratiquent « la plus vieille religion du monde » (La Corée). Pour le fabricant de film, aller rencontrer un chamane, c'est donc aller à la rencontre du passé. Les réalisateurs d'Indo Pino expriment cette conviction d'une manière explicite : « Si [le film] consiste, certes, d'un point de vue géographique, en un voyage dans l'espace, il est essentiellement un voyage dans le passé : il nous ramène aux pratiques médicoreligieuses qu'ont connues probablement dans leur première histoire beaucoup de civilisations, y compris la nôtre » (op.cit.). Avancer dans la jungle, c'est exactement remonter le temps, pour retrouver un degré de l'humanité antérieur au début d'une évolution. C'est un sentiment particulier partagé par l'équipe Cousteau en route vers la demeure d'un chamane jivaro : " Un grand honneur nous est fait : nous sommes invités chez un chamane légendaire d'Amazonie. C'est une sorte de voyage dans le passé.» L'Occident vit dans le présent, il est au terme de l'évolution historique de l'humanité. Le chamanisme, lui, ne peut avoir d'histoire puisqu'il est un phénomène éternel et universel. Il est l'état de tout homme qui n'a pas rompu le cordon ombilical l'unissant à la nature. Le chamane est comme le fossile vivant d'un état de l'humanité antérieur à son entrée dans l'Histoire.

C'est en raison de cet affleurement du passé dans le présent que la rencontre avec le chamane est proprement bouleversante pour l'explorateur. "Le fait que [les rites chamaniques] puissent encore survivre de nos jours rend leur étude extrêmement émouvante et le contact avec ceux qui les pratiquent d'autant plus attachant. D'où les liens très étroits qui se sont tissés entre ces chamans et nous-mêmes au fil des ans » (ibid.). Le chamane est le témoin d'une époque lointaine où l'humanité partageait des représentations religieuses communes. C'est ce qu'illustre le travail de l'Estonien Mark Soosaar, Trois chamanes. Les trois personnages comparés dans le film et qui sont tous trois désignés sous le nom de chamane sont le héros d'une légende estonienne, un chef de tribu séquoia (Amazonie) et un Khante (Sibérie). Il n'y a pas de limites spatiales ou temporelles à l'aire où peuvent se manifester les apparitions de la figure du chamane, si ce n'est celles de la modernité occidentale dont elles sont exclues par définition.

41 Il était prévisible que l'on en vînt à supposer aussi des chamanes aux hommes préhistoriques, les premiers de l'humanité. Cette hypothèse est défendue dans Les chamanes de la préhistoire, un épisode de la collection "Exploration planète " de la Cinquième. Pour le préhistorien Jean Clottes, les auteurs des peintures rupestres sont des chamanes en transe qui ont cru voir des animaux dans les formes des parois des cavernes et ont donné corps à leurs visions en les peignant. L'idée de transe est soutenue à l'image par des plans bougés en surimpression sur les images des parois rocheuses. On a fait venir des Inuits dans les grottes préhistoriques de l'Ariège afin de connaître leurs réactions, et leurs éventuelles explications, face aux peintures rupestres. Survivants d'une humanité disparue depuis longtemps en France, les Inuits devaient pouvoir expliquer le sens de dessins et de formes devenus incompréhensibles pour nous, en raison d'une évolution dont ils ont été préservés.

D'autres civilisations, moins lointaines, ont laissé les traces de l'activité créative de leurs chamanes. Le film L'homme-oiseau : les esprits, l'or et le chamane a été commandé par la Réunion des musées nationaux pour accompagner une exposition au Grand Palais sur 
l'Amérique précolombienne. « Parmi les 55000 pièces que possède le Musée de l'or de la Banque de Colombie, 300 ont été sélectionnées pour être exposées aux regards des Parisiens. Leur point commun? Quelles que soient les époques ou les régions, toutes ont été utilisées par des chamanes pour passer d'un monde à l'autre, pour se transformer qui en jaguar, qui en oiseau» (résumé producteur). Afin de mieux connaître la signification des objets précolombiens, le réalisateur est allé interroger des chamanes amazoniens contemporains chez les Koguis de la Sierra Morena de Santa Marta : là encore, le chamanisme n'est pas quelque chose qui puisse évoluer, puisqu'il se situe avant toute évolution.

Il est notable que des autorités scientifiques sont très souvent parties prenantes de ces élaborations mythiques. Le C.N.R.S. fournit les moyens de production en association avec des chaînes de télévision, tandis que les ethnologues sont conseillers scientifiques, auteurs du commentaire, parfois même réalisateurs, ou bien leurs réflexions font la matière de "bonus " pour les éditions en DVD. On est loin d'un partage des tâches strictement défini entre le savant et le journaliste, tel que Roland Barthes le percevait au sujet de la représentation des "Nègres", et qui lui faisait dénoncer un "divorce accablant de la connaissance et de la mythologie » ([1957] 1970, p. 63). Avec confiance, il affirmait: " La science va vite et droit en son chemin; mais les représentations collectives ne suivent pas, elles sont des siècles en arrière [...] » À l'époque de Barthes, dans la production culturelle populaire, "le Nègre fait peur, il est cannibale " (ibid., p. 62). Aujourd'hui le discours s'est inversé, le journalisme idéalise l'exotique et met en scène ce que Marc Augé (1979) appelle un "évolutionnisme pessimiste ", où c'est le Blanc qui est devenu le cannibale, destructeur des cultures qu'il domine. L'autorité savante est désormais à la traîne et rattrape comme elle peut la course à la surenchère dans l'idéalisation.

La mise en présence de cette figure du passé qu'est le chamane avec la modernité donne lieu naturellement à de douloureuses confrontations. Au milieu de la ville, le chamane apparaît dans tout son archaïsme. C'est au moment de son entrée dans Irkoutsk que l'on comprend que le Russe Dimitri (Chamane) est sans doute devenu luimême un chamane. Il erre perdu, totalement étranger à la vie des habitants, à leurs commerces stériles. Dans Molom, le vieux chamane vagabondant dans la gare d'OulanBator demande un renseignement à un passant vêtu à l'occidental. Celui-ci le repousse en faisant le geste de quelqu'un qui veut montrer qu'il est pressé. Opposé à la démarche lente du chamane, ce geste exprime toute la contradiction entre le monde serein du chamane et la vie affairée et absurde de la ville.

Les boîtes de nuit font peser comme les gares une sourde menace sur les chamanes. Les fabricants de film entretiennent une défiance marquée à leur égard, et les considèrent comme le lieu de perdition par excellence pour l'âme indigène. Dans Le voyage de Charlie, au moment où Charlie dit qu'il est «à la recherche de notre identité spirituelle ", on passe des plans d'insouciants fêtards dans une discothèque. Ces images inquiétantes sont encadrées de plans montrant Charlie rêveur dans la toundra enneigée. Même danger inexorable pour les Évenks : dans le film Taïga terre des chamans, les images d'un endroit semblable ont été choisies pour illustrer une constatation lugubre en off : "Nous avons oublié toutes nos traditions, tout. »

Barthes analysant le film Continent perdu, grand documentaire sur l'« Orient », décrit un imaginaire français qui tend à niveler des contrastes trop effrayants, et s'efforce de démontrer que : «Orient et Occident, tout est égal, il n'y a que des différences de 
couleurs, l'essentiel est identique, qui est la postulation éternelle de l'homme vers Dieu, le caractère dérisoire et contingent des géographies par rapport à cette nature humaine, dont le seul christianisme détient les clefs » ([1957] 1970, pp. 153-154). Dans l'exposition parisienne La grande famille des hommes, Barthes ne voit réaffirmé qu'un même postulat rassurant : "L'homme naît, travaille, rit et meurt partout de la même façon" (ibid., p. 162). C'est assez pour nous permettre de mesurer l'évolution des intentions et des stratégies de nos entrepreneurs culturels depuis les années 1950. Si l'exotisme a toujours cours, ce n'est plus pour affirmer l'unité de l'âme humaine et ses "prédispositions chrétiennes" (ibid., p.153), dont la découverte serait tout à fait ennuyeuse et navrante, mais au contraire pour exalter l'éloignement, l'étrangeté, la différence incommensurable de l'Autre. C'est dans la mesure même où il défie nos usages, échappe à notre histoire et contredit notre philosophie que nous aimons le chamane. Mais s'il en restait là, le chamane ne serait qu'une figure toute négative, or il est aussi un guide, un maître spirituel, endossant une positivité qui reste ambiguë, puisque ce qu'il enseigne, ce n'est jamais que la théorie de sa propre négativité.

\section{Le guide spirituel}

Tu traverseras la noirceur de ton esprit, et si alors tu acceptes tes peurs, tu sauras qui tu es

(Blueberry)

\section{Le sage}

"Les chamanes guident le voyageur", c'est un trait important, méconnu de l'ethnographie, dont nous informe le commentaire du film Les nomades du Grand Khan. Les chemins de son pays lui sont tous connus, il ne peut s'y perdre. Le thème entier de Molom est le voyage d'un petit garçon sous la houlette d'un vieux chamane à travers tout le pays mongol. Plus précisément le chamane est un guide spirituel. «Guidé par mon nouveau maître, je découvrais le sens de la vie », raconte le jeune Yönden en voix off. Le chamane le prévient : "Tu dois apprendre les clefs de la connaissance » et lui promet: "Je t'apprendrai tout». Le chamane rit beaucoup et, semblable au sage oriental, emploie un langage imagé : "La souris va-t-elle attendre le gros ours?» (Chamane), "Si tu es triste, regarde la perle qui brille en toi » (Molom), «Comme la marmotte, ne te fie jamais aux apparences » (ibid.).

D'une manière générale, on doit constater que la figure du chamane est très fortement influencée par celle du sage bouddhiste, dont la présence est bien plus ancienne dans les représentations occidentales. La contamination est particulièrement apparente dans Molom, où le chamane va jusqu'à confier son fils adoptif aux moines d'un temple bouddhique pour achever son éducation. Dans le reportage Spiritualité: chamane à Moscou de Canal Plus, un rite autour d'un feu de bois dans la taïga enneigée montre des " chamanes sibériens » dansant la farandole sous des costumes lamaïques incluant des masques en papier mâché coloré. De tels tropismes affectent certainement depuis longtemps la figure du chamane dans l'imaginaire occidental, comme en témoigne la définition du mot « chaman » donnée par Littré : « Nom de prêtres bouddhistes chez les tribus qui occupent le Nord de l'Asie. » 
49 Le chamanisme n'est pas un phénomène social propre à un groupe, c'est un substrat présent en chaque homme, mais que certains peuples ont su ne pas ensevelir. Le chamane enseigne une technique à ses disciples qui leur permet de réveiller ce substrat latent. Le film occidental exploite beaucoup la relation maître-élève qui permet de mettre en valeur la sagesse du chamane, en tant que Tout-Autre, et l'ignorance du disciple. La relation de domination colon-colonisé, ou Blanc-Sauvage est ainsi inversée. Le film Chamane raconte l'itinéraire spirituel d'un Russe qui cherche à suivre l'enseignement de ceux qui, précisément, sont dominés et persécutés par ses compatriotes: les chamanes. Cette structure narrative rappelle fortement celle $\mathrm{du}$ grand film Dersu Uzala où un Sibérien rieur devenait le guide et puis le maître spirituel involontaire d'un Blanc. Mais le Sibérien était un simple chasseur, et il ne pouvait opérer sur le Blanc la révolution spirituelle que le chamane Anatoli déclenche dans l'âme de Dimitri. L'histoire racontée dans Le voyage de Charlie est, quant à elle, explicitement celle d'une initiation. Le chamane Guillermo dit à son disciple inuit Charlie: "Peu à peu, tu vas apprendre à t'estimer toi-même et donc à estimer les autres. » La chamane Asiri (Les Aïnus de Hokkaido, le jardin où s'amusent les dieux) est allée jusqu'à ouvrir une école dans sa maison, où elle donne des cours de langue et de " philosophie aïnoue ». Les Aïnous assimilés du Japon sont invités dans des stages d'été à renouer avec leurs racines.

Il peut arriver que la quête de l'identité s'applique au chamane lui-même, ce qui semble en contradiction avec sa fonction de connaisseur et gardien des traditions. Seul le documentaire Les nomades du Grand Khan, décidément audacieux, s'est risqué dans ce paradoxe, puisque l'on y fait dire à la comédienne jouant le rôle de la chamane : «J'ai toujours été élevée dans la plus pure tradition chamanique, et je suis partie à la recherche de ce savoir à travers le pays mongol.» Dans cette phrase, on voit deux éléments mythiques se heurter : le chamane comme réceptacle de la tradition dans sa plus grande "pureté ", et le chamanisme comme quête, recherche, ce qui devrait impliquer une ignorance de départ. C'est une contradiction qui se retrouve en général dans les représentations sur le chamane, par exemple chez Michael Harner. Le lecteur de Harner est invité à inventer lui-même les techniques de son chamanisme au cours d'une quête de soi, alors que les exemples qu'on lui propose dans des sociétés exotiques sont, bien entendu, fixés par une tradition où l'improvisation elle-même est normée.

\section{Le dispensateur de bonne humeur et du bien-être}

51 Les apparitions du chamane dans les films occidentaux font voir en général un être avenant, volontiers porté à la bonne humeur, et même aux fous rires. Ces déchaînements de gaieté sont cependant l'exclusivité des films de fiction (Chamane, Molom). Les éclats de rire du chamane surprennent son interlocuteur qui n'en comprend pas la cause, pas plus que le spectateur. Ils mettent en évidence la sagesse supérieure du chamane. Sans aller toujours jusqu'à la franche hilarité, le chamane est en général un homme sympathique. Le commentaire du documentaire Les deux fils $d u$ chamane insiste sur le caractère sociable de Medsinu qui sera finalement élu, à l'inverse de son frère, présenté comme solitaire et peu aimable, et donc naturellement exclu de l'héritage. En Amazonie, le chamane Guillermo Arevalo qui reçoit l'Inuit Charlie (Le voyage de (harlie) apparait comme un personnage cordial, accueillant et aimant faire des 
plaisanteries à l'intention de son hôte. De très gros plans sur son visage avec une faible profondeur de champ mettent en valeur son sourire et ses yeux pétillants.

Le film de cinéma Atanarjuat constitue cependant une exception de ce point de vue. Le mal, à l'origine du drame raconté par le film, a été apporté par un chamane inconnu qui arrive dans la région d'Igloolik et divise une petite communauté d'Inuits. Pour expliquer cette exception, il faut d'abord remarquer que le scénario de ce film a repris les bases d'une légende indigène. Cependant cet écart par rapport à la norme est compensé par l'introduction dans l'histoire de bons chamanes qui, à la fin du film, après avoir défait les forces maléfiques, prêchent à la communauté l'amour et le pardon. Sur ce point le scénario n'a pas suivi la légende ; antérieure à la christianisation des Inuits, elle se terminait par une vendetta sanglante, comme le signale l'anthropologue Bernard Saladin d'Anglure associé à ce film. Une telle fin fut jugée trop cruelle pour le public occidental, et, faut-il croire, peu conforme à notre mythologie du chamane.

Affable et joyeux de caractère, le chamane apporte le bien-être et la santé aux membres de son groupe. C'est une fonction citée dans la quasi-totalité des films consultés : la fonction thérapeutique. Le ou la chamane est " guérisseuse mystique " (Les nomades $d u$ Grand Khan) ou " guérisseur " (Animisme et chamanisme), ou «médecin " (Les deux fils du chaman, Xingu, le corps et les esprits). La transe lui permet d'entrer en contact avec les esprits et, grâce à leur aide, de guérir les malades. Certaines scènes de soins filmées en plein jour dans Le voyage de Charlie expriment cette thèse en faisant subir aux images un traitement semblable à celui des séances chamaniques. Le son direct est remplacé par la musique d'une contrebasse. Le reportage de Canal Plus intitulé Spiritualité: chamane à Moscou s'achève par un concert de musique techno dans une boîte de nuit moscovite, où le chamane Albert est la vedette. À la sortie du concert, un plan américain montre une jeune fille russe se précipitant pour embrasser le chamane, avec ces paroles reconnaissantes : "C'était le pied! ${ }^{20}$ J'étais dans une humeur noire aujourd'hui. Merci beaucoup!» Albert, dont le résumé du producteur annonçait: "Ce chamane-là ne manquera pas de vous surprendre», demeure, en dépit des apparences, fidèle au modèle du chamane qui résout les problèmes psychologiques des autres et répand le bien-être.

54 Le chamane prodigue ces soins par pur dévouement et, d'une manière générale, il n'est pas un être intéressé. Dans Molom, on le voit à Oulan-Bator vendre sa longue-vue en laissant généreusement à l'acheteur le soin de fixer le prix ("À votre convenance »). Il ne jette même pas un coup d'œil au nombre de billets que celui-ci lui donne. À la fin du documentaire Les nomades du Grand Khan, la chamane s'en va car « elle doit rejoindre ceux qui l'ont appelée à leur aide ». Dans Les deux fils du chaman, Medsinu soigne toute la communauté. Sa seule activité consiste à faire le tour des habitations dans la forêt, au prix de longues heures de marche. Il ne semble rien recevoir comme rétribution. Mme Bayar, la chamane d'Oulan-Bator, ne parait pas non plus être rétribuée en échange des services chamaniques qu'elle rend dans la ville. On voit pourtant quelques instants dans le coin du cadre deux personnes pour lesquelles elle fait une divination toucher leur porte-monnaie. Mais le commentaire mystique ne donne aucun éclaircissement sur ce geste. C'est que, comme le souligne le commentaire de Message d'un monde perdu, "le chamane authentique ne tire aucun profit de ses pratiques".

D'une manière générale, dans l'ensemble des films que nous avons pu consulter, des fictions aux documentaires ethnographiques, aucune mention n'est faite des revenus 
ou des moyens de subsistance du chamane, ce qui est caractéristique d'une figure mythique. La chamane du film Les nomades $d u$ Grand Khan vit totalement seule dans une tente conique. Elle n'a pas de bétail pour se nourrir ni même de famille pour l'aider. C'est un être abstrait.

\title{
Le symbole de l'identité ethnique
}

\author{
Les chamanes sont l'esprit \\ et la mémoire d'un peuple \\ (Les nomades du Grand Khan, commentaire)
}

\section{Le représentant de la morale traditionnelle} un jour du cheval d'une petite fille. Le chamane le gronde en lui apprenant que : « Dans la steppe, il ne faut jamais prendre le cheval d'un autre.» Quand l'enfant est mutin, le chaman lui tire l'oreille, mais sans lui faire trop de mal. Le rôle moral du chamane est également présenté dans Les deux fils du chaman, où on le dit "juge ». À un jeune homme infidèle, source de bien des drames dans la vallée, le chamane déclare : « Tu ferais mieux de rester fidèle à ta femme et de suivre mes conseils. » Fidélité conjugale, respect de la propriété privée, mais aussi pardon des offenses, sur ce plan la morale chamanique ne présente pas beaucoup d'exotisme par rapport à la nôtre.

\section{Le chef charismatique}

"Quand on demande aux élèves d'extraire l'essence des fleurs, Alma pense toujours à Touking, le chamane de sa vallée, qui connaît le secret des plantes qui guérissent. " Cette phrase commente un plan où la jeune Alma est occupée à une séance de travaux pratiques en classe (Les deux fils du chaman). Le commentateur affirme ici l'importance que détient le chamane dans le cœur des membres de son groupe, qui sont censés penser à lui à chaque instant. (On observe aussi, mais le fait est courant, que le commentateur est capable de pénétrer la pensée de l'indigène sans avoir besoin de l'interroger.)

Dans ce film, le chamane, comme un chef de village, «veille sur les siens ». Alors que l'image montre une hutte où il est enterré, le commentaire explique que « Touking a exprimé le désir de se faire enterrer dans cette hutte, où il venait régulièrement s'isoler, pour y méditer sur l'avenir de sa communauté ». Cette fois-ci, c'est même $a$ posteriori que le commentateur prend connaissance du contenu des méditations de l'indigène; le sens final en est le même que pour Alma: le chamane est un chef charismatique. Dans la hutte, le cadrage serré, psychologique, insiste sur les pleurs et les lamentations des indigènes auprès de la dépouille du chamane.

\section{Le défenseur de l'identité culturelle}

Le chamane est le garant du maintien des traditions et de la perpétuation de la culture de son groupe, et parfois même un personnage actif en lutte contre les menaces d'évolution, les contaminations extérieures. Dans de très nombreux cas, la présentation de la figure du chamane est accompagnée de descriptions dramatiques sur le sort de la culture de son groupe. Peu sensible au charmes du métissage, le chamane monte la 
garde autour d'une culture essentiellement fermée, dont la survie dépend de son isolement. À la fin du film Les deux fils du chaman, Medsinu a succédé à son père, et le commentateur conclut avec une satisfaction mêlée d'inquiétude: «Un nouveau chamane veille sur les siens. Mais jusqu'à quand Medsinu pourra-t-il être le garant des traditions? Sera-t-il assez fort pour éloigner les dangers de la ville qui attirent les jeunes hommes palawan hors de leur forêt?»

La notion d'« identité » - ou " âme » (Le voyage de Charlie) - demeure assez confuse, mais il semble que l'on puisse la définir comme une substance propre au groupe, qu'il tient du passé et qui se transmet par le sang de génération en génération, consciemment ou inconsciemment. Quand bien même les Inuits dans Le voyage de Charlie ont été sédentarisés et christianisés, et en dépit du désintérêt complet des jeunes générations pour le chamanisme, leur « identité profonde » demeure en réalité la culture qui a été celle de leurs ancêtres. La continuité par le sang justifie la stimulation d'une réinvention de la tradition organisée non seulement par les producteurs du film, mais par des anthropologues associés. Grâce à l'idée des scientifiques et au financement du producteur du documentaire, Charlie reçoit une initiation chamanique et la possibilité d'assumer lui-même le rôle du chamane dans sa communauté. Or, si le film est réussi, il demeure que le mythe qui fait rêver les Occidentaux n'est pas encore partagé par les Inuits. C'est ce dont témoigne l'aveu de l'anthropologue Bernard Saladin d'Anglure, déjà intervenu dans Atanarjuat, et qui était à l'origine de ce projet : « [Après le voyage] Charlie avait pensé un moment devenir chamane, mais il ne veut à aucun prix se couper de sa communauté qui est anglicane. " Chez les Inuits, recréer l'« identité culturelle " en se faisant chamane, ce serait donc s'exclure de sa communauté et non en devenir le chef charismatique comme le voulait le mythe occidental.

D'après les représentations occidentales, la transmission de la culture par le sang dans les sociétés à chamanes semble être un fait général. Pour les Aïnous du Japon, le commentateur du film Les Aïnus de Hokkaido explique sur un ton mélancolique : «Les générations se sont entremêlées. Leur sang s'est mélangé, ils ont perdu leur identité. Dans les années soixante il ne restait plus que trois cents Aïnous de pure race dans le monde. » D'après ces propos, la perte de la pureté de la « race » est directement liée à la disparition de l'identité culturelle. Aussi la chamane Asiri fait-elle œuvre utile en réunissant autour d'elle des personnes de culture japonaise mais ayant un ancêtre aïnou, afin de leur faire retrouver leur vraie culture, leur « identité ».

\section{Le gardien de la primitivité}

Le chamane est l'image de l'humanité apparaissant dans sa primitivité. Les pratiques du chamane sont insérées dans un ensemble de comportements et de visions du monde qui appartiennent bien plus à l'état de nature qu'à l'état de culture. Voici un extrait du résumé d'Amazonie: les derniers Indiens fait par le documentaliste de l'INA: «Nous voyons de nombreux plans de ces sorciers en train d'absorber de la drogue ce qui les met littéralement en transe. Également de nombreux plans de femmes et d'enfants. On voit par exemple des enfants s'épouillant et mangeant avec délectation les animaux trouvés dans les cheveux de leur camarade. Autre image forte : un indien apprend à gratter une allumette. » Ignorant à la fois les interdits de la Culture (on ne doit pas manger d'insectes) et ses inventions techniques (les allumettes), les «derniers» Indiens sont définis par rapport aux goûts et dégoûts de l'Occident. Ce que les 
commentateurs appellent culture indigène se construit toujours négativement. Leur culture, c'est d'être à l'écart de la Culture, et s'ils sont chamanistes, c'est que tout homme l'est par défaut.

Le magazine pédagogique "Exploration planète ", dans son document sur Les chamanes de la préhistoire, donne la définition suivante: "Le chamanisme peut être considéré comme une religion universelle que pratiquent encore certaines tribus aujourd'hui. » Seuls certains groupes sont parvenus à protéger ce fonds " encore " jusqu'à nos jours ; ce privilège est souvent signalé sur la bande-son par l'usage de musiques reconnaissables. Signe que la primitivité est un phénomène universel, les musiques de percussion africaines peuvent être utilisées aussi bien pour des hommes préhistoriques (Les chamanes de la préhistoire), que pour les Évenks (Taïga, terre des chamans). Les fabricants du film Atanarjuat ne font-ils pas résonner les chants de montagnards touvas du groupe Huun Huur $\mathrm{Tu}^{21}$ sur les images de la toundra canadienne ? Les exemples de ces imprécisions précises et calculées sont innombrables.

Une fraternité mystérieuse unit les peuples restés longtemps isolés - les "peuples autochtones " (Le voyage de Charlie), désignation obscure quand elle est utilisée ainsi dans l'absolu et à l'échelle de la planète, et non par opposition à des populations immigrées, et qui ne se substitue qu'avec peine à l'irremplaçable terme "peuples primitifs ». Le réalisateur du Voyage de Charlie s'interroge : "Existe-t-il au delà des différences de traditions des valeurs trans-ethniques? Aujourd'hui des peuples autochtones s'organisent pour lutter face à la globalisation qui menace leurs cultures. C'est dans ce contexte que s'inscrit l'histoire de Charlie. » ${ }^{22}$ La question est purement oratoire, car Stéphane Bégoin y a déjà répondu positivement en réalisant son film. Avec des amis anthropologues, Bernard Saladin d'Anglure et son épouse Françoise Morin, il a fait parvenir à Charlie une cassette vidéo dans laquelle Guillermo, le chamane shipibo, dit à l'Inuit: "Charlie, je t'invite chez nous » (cette séquence, mise en scène pour la caméra, se situe au début du film). Dans son voyage, Charlie reconnaissant se dit « très content de rencontrer mes frères autochtones ». Françoise Morin le fait inviter dans le studio d'une radio locale des Shipibo dont le speaker présente Charlie comme « un de nos frères indigènes ». Du début à la fin, la fraternité indigène est suscitée et orchestrée par les Occidentaux. Et il ne peut en être autrement, puisque les indigènes ne sont « indigènes ", " primitifs ", ou «Autres " que du seul point de vue de "Nous », les indigènes de l'universel, qui les englobons et les réunissons dans une même collectivité. Les représentants des différents peuples «indigènes » sont capables de communiquer entre eux, par-delà la barrière des langues et des cultures. Le montage du documentaire Le voyage de Charlie supprime toutes les apparitions visuelles ou sonores de l'interprète. L'Inuit (francophone) et le Shipibo du Pérou (doublé en français) dialoguent librement dans la langue de Molière, en un champ-contrechamp de gros plans sans profondeur de champ, parfaitement nettoyé de tout tiers externe. Le contexte est expulsé, seule demeure la mise en scène de deux individus, frères dans la primitivité, dont l'un va initier l'autre à son « identité profonde ».

Le miracle de la communicabilité universelle peut aussi s'étendre à ceux qui ont réussi à se laver des atteintes de la civilisation occidentale. Peut communiquer directement avec le chamane l'Occidental juste ou purifié, dont la figure la plus favorable est l'artiste : c'est le cas du Russe Dimitri, violoniste virtuose (Chamane). Dans une séquence surprenante, la mère d'Anatoli, vieille chamane, parle en iakoute à Dimitri qui la comprend et lui répond en russe. Un peu plus tard dans la nuit, Dimitri, aidé par une 
boisson psychotrope, fait l'expérience des représentations chamaniques : il voit des animaux empaillés s'agiter et grogner autour de lui. Comme Dimitri, les vidéastes, plasticiens et photographes rassemblés dans l'exposition Yanomami, l'esprit de la forêt étaient capables de percevoir directement les images mentales de chamanes appartenant à un peuple dont, selon leur propre aveu, ils ignoraient l'existence peu auparavant, ou qu'ils n'ont jamais rencontré. Pour cela certains d'entre eux ont utilisé comme Dimitri l'aide d'une substance hallucinogène locale, tandis que d'autres se sont contentés de laisser libre cours à leur imagination. On ne peut mieux affirmer le caractère infra-culturel du chamanisme.

\section{La victime agonisante de l'Occident}

67 Le chamane subit depuis longtemps la répression de l'Occident au point de se trouver aujourd'hui dans un état inquiétant; comme le chamanisme et les cultures persécutées qu'il représente, il est moribond. Souvent mélancoliques, beaucoup de films se concentrent sur la mort du chamane. Le voyage de Charlie commence par l'annonce de la mort de Victor Tungulik, le dernier chamane inuit: «Avec Victor Tungulik disparaît une pratique ancestrale qui était au cœur de la vie des Inuits. " À la fin de Molom, le chamane mongol, vieillard voûté, se dirige à pas lents vers la mort. L'histoire du film Les deux fils du chamane est le deuil d'un village qui a perdu son chamane et peine à lui trouver un successeur.

Comme son nom l'indique, le documentaire Death of a shaman est également consacré à ce thème. Ce film fait le portrait d'un chamane d'origine thaïlandaise vivant avec sa famille aux États-Unis, où il est confronté « à la pauvreté, au racisme, aux religions, aux drogues, à la prison, à la violence ». À travers le destin cruel du chamane, c'est l'exil de la communauté thaïlandaise qui est illustré, et son "identité perdue ${ }^{23}$, comme l'explique la présentation du producteur. L'auteur du film, Fahm Saeyang, qui n'est autre que la fille du chamane, montre un homme désespéré par une Amérique qui a trahi ses rêves. Ce sont finalement la drogue et la dépression qui le tuent en novembre 2000 au cours du tournage. Tentée à ce moment de tout abandonner, la jeune femme fut encouragée par son coréalisateur, Richard Hall, à prolonger son travail et à mettre en valeur au contraire le thème de la mort de son père. L'Américain connaît, mieux que la jeune femme immigrée, la puissance symbolique de la mort d'un chamane dans la mythologie occidentale.

69 Le persécuteur, s'il est nommé directement, emprunte en général son visage à trois figures possibles : le missionnaire chrétien, le Soviétique ou le chercheur d'or. Il est à noter que la répression dont le chamanisme a pu être victime sous l'action de prosélytismes non occidentaux ne retient guère l'attention. Nous n'avons rencontré qu'un documentaire sur la Kirghizie pour souligner l'opposition entre l'islam et le chamanisme en Asie centrale. Quant à l'action du bouddhisme lamaïque, bien plus importante que celle du christianisme pour des zones comme la Mongolie et le sud de la Sibérie, elle n'est pas mentionnée. Au contraire, Molom fait du chamanisme et du bouddhisme les deux facettes, l'une rurale, l'autre urbaine, d'une seule et même pensée (c'est le chamane qui initie le jeune garçon au culte du Bouddha).

Chamane et Taïga terre des chamans illustrent avec précision, l'un dans la fiction, l'autre dans le documentaire, l'écrasement du chamane par le système soviétique - dispositif censé trouver dans la Russie contemporaine un prolongement fidèle. Dans le film de 
Bartabas, le chamane iakoute est incarcéré dans un goulag. Il y subit les brimades racistes des Soviétiques qui l'appellent "face de singe » et le brutalisent. Après son évasion, il est poursuivi par les surveillants du camp qui lui tirent dessus, en même temps que sur son compagnon, fuyant à cheval. Finalement, il connaît une mort pathétique. On le voit d'abord vaciller sur sa selle, puis tomber dans la neige avec un trou rouge dans le dos. Un long plan moyen, composé comme une admirable pietà, montre ensuite l'agonie du chamane allongé à terre avec Dimitri à genoux près de lui, lui tenant la main. Le thème de la persécution de chamanes dans le film Taïga, terre des chamans est associé à celui de la destruction que les Russes imposent à la forêt. Des plans en contre-plongée et contre-jour donnent une vision épouvantable des pelleteuses des mines d'or, image soutenue par une musique effrayante et un grondement de moteur d'avion. Le sort des Évenks est lié à celui de la forêt, mais aussi à celui d'un peuple victime de la répression russe, emblématique pour le public occidental : les Tchétchènes. Un jeune Évenk, Aliocha, est mort en Tchétchénie au cours de son service. D'après le commentaire, c'est des Russes qu'il a, au fond, été la victime et non des Tchétchènes qui l'ont tué. Un instituteur affirme près de sa tombe : «Nous, le peuple évenki nous pensons que cette guerre est injuste et nous sommes d'accord avec le peuple tchétchène. » Mais c'est surtout le doubleur qui l'affirme avec chaleur, la voix originale en russe disparaissant juste à ce moment ${ }^{24}$. Une fois évoqué en arrièrefond le drame tchétchène, la plainte de l'ethnographe Anatoli devient très convaincante: "Même aujourd'hui, le chamanisme chez les petits peuples du Nord souffre de l'oppression de l'État. ${ }^{25}$ Que les situations se modifient ou non, que les régimes changent, que les mentalités et les modes se retournent, rien n'y fait: il appartient à la figure du chamane d'être éternellement une victime de l'Occident.

\section{Le rédempteur de l'homme moderne}

71 Pour sa part, le chamane n'est pas un ennemi absolument hostile à l'Occident. La lutte qu'il mène contre lui est une lutte défensive visant à sauvegarder les valeurs qu'il représente. À l'égard des Occidentaux qui font l'effort de venir vers lui, d'écouter son message, il est bon, son action est bienfaisante, voire salvatrice. Dans la fiction de Bartabas, c'est bien le chamane Anatoli qui sauve le Russe Dimitri en lui donnant l'idée de s'évader du goulag, en le menant dans la forêt, en lui donnant un cheval, en le guidant spirituellement ensuite après sa mort. Perdu dans la taïga, Dimitri est recueilli par des éleveurs iakoutes et hébergé chez une vieille chamane, la mère d'Anatoli. Cette histoire en rappelle une autre, dont elle n'est certainement pas inspirée, mais avec laquelle elle présente des analogies de structures révélatrices: il s'agit d'un roman d'Eugène Müller publié en 1860, Un Français en Sibérie. Victime innocente de l'arbitraire du pouvoir tsariste, un Français cultivé en visite à Pétersbourg est emprisonné et relégué sans jugement en Sibérie. Il s'évade et doit sa survie au secours de nomades indigènes. Dans un registre moins dramatique, on voit réapparaître ce rôle de guide de l'Occidental fourvoyé à l'intérieur d'un jeu télévisé de 1986. Les joueurs perdus dans la forêt reçoivent des conseils à la fois pratiques et moraux d'un chamane généreux qui leur permettra de retrouver le droit chemin. Le chamane est auprès des siens un guérisseur, nous l'avons vu; il l'est aussi pour l'Occidental, comme en témoigne le documentaire Iboga, les hommes du bois sacré de Kellner. Ce film analyse l'usage de la drogue iboga chez les chamanes pygmées du Gabon. Selon le commentateur, l'iboga est une «drogue saine ». Un exemple frappant de rédemption est même donné : c'est 
l'histoire d'un héroïnomane venu se faire soigner chez les Pygmées. Grâce aux soins des chamanes et à la consommation de la plante iboga, l'Occidental est parvenu à la guérison: «Il a résolu son problème de dépendance physique et psychologique.» On s'émerveille de constater que les plus frustes sauvages possèdent des méthodes de guérison qui permettent d'obtenir ce que la science occidentale, avec toute sa morgue, désespère d'atteindre. La grande production française Blueberry, malgré son échec commercial, n'en est pas moins une remarquable illustration de ce schéma scénaristique, où le chamane ramène l'ordre, le bien-être physique et intérieur, chez ceux même qui, dans leur aveuglement, le persécutent. La découverte du chamanisme, grâce au chamane indien Runi et à d'utiles plantes psychotropes, permet au shérif Blueberry, non seulement d'affronter et de vaincre le dangereux tueur Blount qui met en péril l'ordre public dans son village, mais aussi «d'affronter ses propres démons intérieurs et de retrouver sa liberté ${ }^{26}$.

Selon la présentation de son producteur, Canal Plus, le reportage Spiritualité : chamane à Moscou «permet de mieux comprendre le lien entre les rites de la transe depuis le début de l'humanité avec la quête moderne de la "generation rave", dont le support est la techno ». Le chamane rockeur vient donner un nouveau souffle à la techno occidentale en l'ouvrant à des valeurs plus profondes. Dans le reportage, le commentateur affirme d'emblée qu'il est nécessaire de "retrouver à travers la musique une spiritualité ; dans le futur il faudra aussi s'occuper de son âme ». Dès lors le chamane n'est plus seulement une figure d'un passé fossilisé. Le passé qu'il représente est chargé de valeurs positives dont l'avenir doit se nourrir. En lui reposent les espoirs de l'Occident rêvant de se racheter.

C'est en effet dans l'enseignement du chamane, fait d'écologisme, de pacifisme et d'antimondialisme, que l'Occident peut se corriger de ses défauts les plus graves. Prenant exemple sur le chamane, l'homme moderne peut retrouver la conscience de l'importance de la nature, de la nécessité de vivre en harmonie avec elle et avec les autres. "La violence ne sert à rien face à la nature et aux hommes qui en font partie " dit Asiri (Les Aïnus de Hokkaido). Aux Japonais d'origine aïnoue qu'elle reçoit dans ses stages de formation, la chamane livre un enseignement valable pour tout le monde moderne: "La société moderne surproduit, jette des aliments et n'hésite pas à y mélanger des produits synthétiques et chimiques, c'est un non-sens. La société d'aujourd'hui est trop riche.» Selon le commentaire, "en venant ici les citadins espèrent retrouver une notion des valeurs qu'ils ont perdues avec la société moderne ». Le fait qu'ils soient Japonais et non Occidentaux n'est pas gênant: ils sont sans difficulté assimilés à l'homme moderne, car les Japonais ne nuisent pas moins que les autres à la nature et aux indigènes, en l'occurrence les Aïnous. Ils bâtissent des barrages qui, montrés à l'image, sont accompagnés par une bande-son effrayante, semblable à celle de la séquence des mines d'or russes de Taïga, terre des chamans. La ressemblance de construction n'est pas un hasard; de fait, la position est la même chez les Sibériens, représentés par l'ethnologue Anatoli: «C'est de la nature que tout provient. Nous devons la préserver, c'est ce que nous apprend le chamanisme, mais nous, hommes modernes, nous détruisons tout, seul compte le fric, le fric, le fric... » L'Occident doit suivre les préceptes salutaires du chamanisme car «si la taïga périt, l'humanité périra avec elle » (Taïga, terre des chamans). Le chamane offre en somme une authentique alternative à la culture occidentale, à la fois un modèle dont elle devrait s'inspirer et la preuve rassurante que l'Autre existe encore, malgré la mondialisation et 
l'uniformisation des cultures qui place l'Occidental dans un vertigineux jeu de miroir. Les autochtones de tous les pays, nous dit-on, s'unissent pour lutter contre la mondialisation, nouvelle forme de l'impérialisme occidental. Mais même cette lutte contre lui-même, c'est l'Occidental qui l'organise (Le voyage de Charlie). L'Occident a besoin de cet "Autre ", incarnation de ses péchés, et dans lequel il place aussi ses espoirs de salut.

On touche là sans doute au paradoxe essentiel et fondateur du mythe du chamane. Le chamane, victime agonisante, incarne les souffrances imposées par l'Occident à l'Autre. Mais le porteur du péché de l'Occident peut aussi être celui par lequel l'Occident rachètera sa faute. Il est en même temps la figure de la rédemption. Une telle incarnation de la rédemption sous la forme d'une victime innocente ne peut, naturellement, manquer d'évoquer une inspiration christique. C'est dans son propre fonds culturel que l'Occident a trouvé les éléments essentiels lui permettant de construire la figure du chamane.

On ne saurait cependant considérer qu'il s'agit là d'une simple assimilation de l'Autre à Soi, comme celle dont Barthes déconstruisait le mécanisme à propos du film Continent perdu. Aujourd'hui, l'Autre est magnifié dans sa différence, cette étrangeté objective qui est un garant de son identité ancestrale. Si la structure narrative de la victime rédemptrice est bien un thème propre à la culture occidentale, le protagoniste nommé " chamane » dans cette fiction est tenu d'être étrange et de porter les marques visibles et intelligibles de son altérité. Que ces marques soient elles-mêmes des rêveries codées de l'Occident, cela ne fait pas perdre sa force au dispositif tant que la signature du code reste discrète. Car réussir un film à chamanes, c'est autant montrer d'une manière nouvelle ce qui était attendu que dissimuler ce qu'on ne saurait voir ; en particulier, c'est cacher qu'on a posé face au chamane un appareil cinématographique, car dès que le chamane regarderait la caméra (dès que le spectateur le verrait le voir), il subirait le contact, il échapperait à son identité immaculée et se trouverait projeté dans l'Histoire. Filmer le chamane conformément au mythe, c'est cacher une intervention et une relation qui s'établit entre notre imaginaire et le sien; à cette condition seule, scrupuleusement respectée par les fabricants de film, l'objectivité de l'étrangeté est préservée, et le mythe peut continuer à accomplir son travail monotone et sourd.

\section{Le mythe et nous}

Il est rare que l'ethnologue amené à analyser les productions mythiques de sa propre société parvienne à garder, s'il le tente seulement, le point de vue impartial et teinté d'une discrète sympathie qu'il adopte naturellement à l'égard de son terrain familier. Le « regard éloigné » s'émeut, se trouble, se fait regard distant, au sens moral cette fois. Le cas sans doute le plus frappant de perte de sang-froid dans l'analyse de mythes occidentaux est constitué par une tentative non ethnologique mais qui se recommande à l'occasion de l'ethnologie, ce sont les célèbres Mythologies de Roland Barthes. Si Barthes ([1957] 1970) affirme se placer « du point de vue de l'ethnologue» (p. 177) afin d'analyser à travers « un beau matériel anthropologique » «l'ethnie française » (p. 92), les termes qu'il emploie - « irritant », stupide » et « condamnable » (p. 60), « technique générale de mystification » (p.127), "représentations [...] stagnantes dans l'erreur » (p. 63), «fragilité mentale de la petite-bourgeoisie française» (p. 94), «bêtise objective" (p.65) - ont plus d'affinités avec les propos des voyageurs semi- 
ethnographes du XIX siècle, tel Raoul Bourdier chez les Tchouktches - «ridicules pratiques de leurs chamans ", "un tissu obscur de superstitions absurdes ", "théorie absurde ${ }^{27}-$, qu'avec les analyses d'un spécialiste des Autres contemporain de Barthes, Lévi-Strauss, pour qui, bien au contraire, dans les mythes, «l'esprit, relativement à l'abri des contraintes externes, déploie encore une activité native qu'on peut observer dans toute sa fraîcheur et sa spontanéité » (Lévi-Strauss 1983, p. 236). Certes il y aurait une illusion épistémologique à adopter la même méthode pour les sociétés exotiques et pour la sienne propre, comme si le fait d'être ou non partie prenante de son objet d'étude était indifférent. Il y aurait surtout une irresponsabilité morale à ne pas reconnaître la différence d'efficacité qui accompagne le mythe occidental et le mythe bororo. Une lecture purement esthétique des mythes liés à la négritude qui ne tiendrait nul compte de leur rôle de légitimation de l'impérialisme colonial serait coupable, venant d'un membre d'une société ayant un passé colonialiste. Les mythes des dominants n'ont pas le même pouvoir que ceux des dominés, surtout quand ils portent sur ces dominés eux-mêmes. Mais entre l'analyse historique de l'implication de certaines mythologies dans la construction idéologique de l'impérialisme occidental et le traitement systématiquement démystifiant et chargé de condamnation morale du mythe occidental en tant qu'occidental, il y a un pas qu'aucune nécessité logique ou historique n'oblige à franchir. En particulier, les représentations fascinées et mythiques de l'Autre précèdent, excèdent géographiquement le colonialisme, et lui survivent. Bien plus, comme on a pu le voir dans cette étude, les productions concernant les chamanes mettent en image un discours dont la teneur politique, quand elle s'exprime, se situe sans ambiguïté dans l'héritage de l'anticolonialisme et poussent le zèle à faire porter la critique sur des sociétés qui n'ont pas subi le colonialisme occidental.

Un mythe, s'il est occidental, sera qualifié par l'Occidental de "mystification retorse " (Barthes [1957] 1970, p. 31), - s'il est exotique, il sera une œuvre d'art. Mais cette différence de traitement n'affirme-t-elle pas une différence de nature entre nos sociétés, qui auraient pour devoir d'être le réceptacle transparent de l'unique vérité scientifique, et d'autres, chargées de garder le souvenir d'un état fantaisiste, magique et archaïque de l'humanité? N'est-ce pas postuler l'existence d'essences humaines différentes qui appelleraient par leur nature des partis pris épistémologiques et moraux opposés?

Les diverses caractéristiques que nous avons rassemblées autour du chamane mythologique présentent des contradictions. Le chamane, par exemple, peut difficilement être à la fois une figure muette et un guide spirituel. Mais la tâche des fabricants de film est précisément de trouver des solutions originales pour résoudre les contradictions internes du mythe: ainsi on pourra présenter un chamane seulement taciturne plutôt que muet, ou bien on confiera son enseignement à un tiers. Certains films, rares, ont cependant assez d'indépendance pour s'écarter du modèle. Mais le seul qu'on puisse dire vraiment indifférent à l'égard du mythe, c'est sans doute le documentaire Chasseurs et chamans du cinéaste Raymond Depardon, qui se contente de montrer à partir d'un point de vue relativement fixe, sans commentaire, effets tendancieux de cadrage ou trucages de post-production, une séance de soin chamanique chez les Yanomamis. Pourtant les journalistes et le producteur en font tout de même une illustration "poétique " de "la transe et ses images mentales". L'interprétation donnée par les critiques fait, elle aussi, partie de la post-production du film, et à cette ultime étape, la figure mythique du chamane peut encore reprendre le 
dessus, contre les intentions du réalisateur qui, en l'occurrence, se disait simple " passeur » ${ }^{28}$, affirmant honnêtement les limites tout autant que la réalité de son rôle de protagoniste dans une relation.

Le chamane représente pour l'Occident le Tout-Autre. Il vit au cœur d'une nature intacte, alors que la société occidentale est urbaine. Il connaît et respecte son environnement, il consomme peu, alors que l'Occident surproduit. Il manifeste l'union avec le cosmos par la transe, alors que l'Occidental instrumentalise les ressources de la Terre. Il est la figure du désintéressement opposée à la société du profit. Il est naturel, alors que l'Occidental vit dans l'artificiel. Il est l'ancien, le préhistorique, alors que l'Occidental est à la pointe extrême de la modernité. Devenant quelquefois une figure active, il est porteur de valeurs écologiques et antimondialistes. Il devient un instrument de lutte politique, au risque d'être transformé en marionnette comme l'illustre le film Le voyage de Charlie.

Le mythe du chamane est celui d'un homme de la nature, persécuté par des Occidentaux répressifs, mis à mort, mais qui donne à l'Occidental juste la chance de sa rédemption. Pourquoi les films rejouent-ils inlassablement cette Passion polyphonique du chamane? Sans doute pour résoudre l'attrait et la répulsion que l'Autre inspire à l'Occident - l'objet du mythe est de fournir un modèle logique pour résoudre une contradiction, affirmait Lévi-Strauss. Mais aussi pour garantir l'existence même de cet Autre dont la survie est de moins en moins visible. (Comme si l'uniformisation des vêtements et des habitats entraînait immanquablement celle des cultures.) Si l'Occident peut s'accuser de le faire souffrir, c'est que l'Autre survit encore quelque part, et qu'il existe donc une autre voie que celle de la vie occidentale. L'effrayant jeu de miroir dans lequel l'Occidental voit son image insipide répétée dans tout l'univers s'interrompt alors pour un moment.

\section{BIBLIOGRAPHIE}

Augé, Marc

1979 Symbole, fonction, histoire, les interrogations de l'anthropologie (Paris, Hachette).

Barthes, Roland

[1957] 1970 Mythologies (Paris, Le Seuil).

Colleyn, Jean-Paul

1993 Le regard documentaire (Paris, Éd. du centre Pompidou).

Grève, Claude de

1990 Le voyage en Russie, (Paris, Robert Laffont) [Bouquins].

Gautier, Guy

[1995] 2002 Le documentaire, un autre cinéma (Paris, Nathan).

Harner, Michael

1982 Chamane, trad. de l'anglais par Zénio Bianu (Paris, Albin Michel) [Expérience intérieure]. 
Lévi-Strauss, Claude

[1973] 1997 Anthropologie structurale deux (Paris Plon) [Agora].

1983 Le regard éloigné (Paris, Plon).

Roche, Denis

1995 La poésie est inadmissible (Paris, Seuil).

Villain, Dominique

2001. L'œil à la caméra, le cadrage au cinéma (Paris, Cahiers du cinéma).

Wittgenstein, Ludwig

[1971] 1992. Leçons et conversations (Paris, Gallimard) [Folio Essais].

\section{FILMOGRAPHIE}

La date indiquée à droite du titre est celle de la production du film, la seconde correspond à la première diffusion. La mention « diff. » signifie diffuseur. « Producteur diff. » signifie que le film a été autoproduit par la chaîne sans coproduction et « commanditaire diff. » que le film a été commandé par le diffuseur. En l'absence de la mention « commanditaire », la diffusion correspond à un achat de droits de diffusion.

Les Aïnus de Hokkaido, le jardin où s'amusent les dieux, 1997.

Documentaire d'Alain Bourillon, 50 min. Prod. UDA, commanditaire diff. La Cinquième, 1998. Asiri, une chamane aïnoue tente de restaurer la culture de son peuple.

Amazonie : les derniers Indiens, 1988.

Documentaire de Christian Brincourt, 26 min. Prod.-diff. TF1, 1988.

La vie quotidienne dans un village d'Amazonie. La consommation de la drogue parika.

Animisme et chamanisme, 1996.

Magazine (« Des religions et des hommes ») de Claude Theret, 13 min. Prod. Vidéa film, commanditaire diff. La Cinquième, 1996.

Émission pédagogique commentée par Jean Delumeau et illustrée de documents d'archives.

Atanarjuat, l'homme rapide, 2001.

Fiction de Zacharias Kunuk, 168 min. Prod. National film Board, Canada. Diffusion cinéma. Drame sur la base d'une légende inuit.

Blueberry, 2004.

Fiction de Jan Kounen, Prod. Thomas Langman et Ariel Zeïtoun, France. Diffusion cinéma. Le scénario allie des éléments de western (courses de chevaux, duels, quête au trésor) à une recherche mystique au cours de laquelle le shérif Blueberry entre en contact de manière spectaculaire avec les esprits de la montagne indienne.

Le chaman et son apprenti, 1995.

Documentaire de Reid Howard, 48 min. Prod. La Sept, Arte, diff. Arte, 1995.

Chamane, 1996.

Fiction de Bartabas, France. Diffusion cinéma.

Le chamane iakoute Anatoli aide le violoniste russe Dimitri à s'évader du goulag. Mais Anatoli est tué au cours de leur fuite. Dimitri doit errer seul dans la taïga avec pour seul guide l'esprit d'Anatoli. Ce voyage est une initiation, à la fin de laquelle Dimitri devient lui-même, en quelque sorte, chamane. 
Les chamanes de la préhistoire, 1998.

Magazine («Exploration planète ») de Pierre Stine, 14 min. Prod. France 2, Gédéon, La Cinquième, diff. La Cinquième, 1999.

Le préhistorien Jean Clottes présente son hypothèse selon laquelle les auteurs des peintures rupestres des grottes préhistoriques étaient des chamanes en transe.

Le chamanisme aujourd'hui, 1997.

Magazine («Archimède »), anonyme à l'INA, 12 min. Prod. ZDF Mayence, diff. Arte, 1997.

Émission pédagogique, illustrée par de nombreux exemples filmés.

Chasseurs et chamans, 2002.

Documentaire de Raymond Depardon, $32 \mathrm{~min}$.

Commande de la Fondation Cartier pour l'art contemporain. Non diffusé. Exemple rare

d'utilisation du cinéma direct pour une société exotique, les Yanomamis. Entre les scènes de chasse et celles de chamanisme, une partie de football au village est filmée en plan-séquence.

La Corée, 1992.

Documentaire de Bernard d'Abrigeon, (Série « Des trains pas comme les autres »), $44 \mathrm{~min}$. Producteur diff. Antenne 2, 1992.

Opposition entre Séoul et son « modernisme agressif » et l'île de Cheju où l'on pratique le chamanisme.

Death of a shaman, 2003.

Documentaire de Richard Hall et Fahm Saeyang. Prix du meilleur long métrage documentaire du festival international d'Oakland. Non diffusé.

Aux États-Unis, la vie pénible de la famille de Fahm Saeyang, dont le père est un chamane d'origine thaïlandaise. Victime de persécutions racistes, il est mort d'épuisement au cours du tournage.

Dersu Uzala, 1974

Fiction d'Akira Kurosawa. Prod. Mosfilm, Moscou. Diffusion cinéma.

Un chasseur sibérien guide une troupe de soldats russes dans la taïga.

Les deux fils du chaman, 1998.

Documentaire de Boccanfuso, 57 min. Prod. Gédéon, C.N.R.S. audiovisuel, diff. Arte, 1998. Avec le soutien du ministère de la Culture, et du ministère de l'Éducation nationale.

Chez les Palawan, dans les Philippines, le chamane Touking vient de mourir. Alma qui fait ses études en ville rentre au village. Là, l'un des deux fils du chamane va devoir lui succéder. C'est le charismatique Medsinu qui l'emportera.

Les enfants de la lune: Indiens d'Amazonie, 1984.

Documentaire de Pierre Bouhin. Prod. France régions 3, Lyon, PRD Riviera films, commanditaire diff. FR3, 1986.

Cérémonie d'initiation d'un nouveau chamane chez les Yanomamis d'Amazonie.

L'homme-oiseau : les esprits, l'or et le chamane, 2000.

Documentaire de François Fronty, 51 min. Prod. La Cinquième, Réunion des musées nationaux, Les films du tambour de soie, diff. La Cinquième, 2000.

Les objets du musée de Bogota présentés dans l'exposition du Grand Palais « Les esprits, l'or et le chamane ", confrontés à des chamanes contemporains d'Amazonie.

La forêt, 1985.

Création originale d'Yves Gautier, 13 min. Prod. PRD, Paris, commanditaire diff. TF1, 1985. 
Une petite fille se promène dans la forêt et rencontre d'étranges personnages, les chamanes.

« Reportage en forme de fiction» (commentaire de l'INA).

Les grands pêcheurs de la côte Nord-Ouest, 1983.

Documentaire de Thérèse Patry, 52 min. Prod.-diff. TF1, 1983.

La pêche au saumon, encadrée de cérémonies chamaniques.

Himalaya, l'enfance d'un chef, 1999.

Fiction d'Éric Valli, 104 min. Prod. Jacques Perrin, France. Diffusion cinéma.

Au Tibet, contre les avis du chamane, une caravane de sel se lance dans la montagne.

Iboga, les hommes du bois sacré, 2002.

Documentaire de Gilbert Kellner, 53 min. Prod. Art Line Films, Paris, commanditaire diff. France $5,2002$.

Au Gabon chez les Pygmées, enquête sur la drogue saine iboga.

Indo Pino, 2002.

Documentaire de Martine Journet et Gérard Nougarol. Prix Nanook au 21 ${ }^{\mathrm{e}}$ Bilan du film ethnographique. Non diffusé.

Indo Pino, une chamane wana d'Indonésie tombe malade. Les réalisateurs lui donnent des antibiotiques, qu'elle intègre à son système de représentations.

Message d'un monde perdu, 1984.

Documentaire de Jacques-Yves Cousteau, $48 \mathrm{~min}$. Prod. Cousteau Society Incorporation, E.U. Film diffusé 37 fois entre 1988 et 2000 sur TF1 et Antenne 2.

Les équipes Cousteau partent à la découverte de l'Amazonie et des Indiens Jivaros.

Molom, conte mongol, 1995.

Fiction de Marie Jaoul de Poncheville, 96 min. Diffusion cinéma.

Molom, un chamane descendu du ciel trouve un enfant sauvage abandonné parmi les loups, Yönden. Il prend en charge son éducation, et le mène finalement dans un monastère bouddhique.

Les nomades du Grand Khan, 1998.

Documentaire de Dimitri Grimblat, 53 min. Prod. BFC, Paris, commanditaire diff. La Cinquième. La Mongolie à travers le portrait d'une chamane et de ses deux frères, l'un éleveur nomade, l'autre citadin.

Oulan-Bator, 1999.

Documentaire de Xavier Simon, 43 min. Prod. Les Films d'ici, Paris, commanditaire diff. La Sept, Arte, 1999.

La ville d'Oulan-Bator, racontée par l'esprit errant d'un cheval : la chamane Mme Bayar, le lama, le travail à l'usine, les enfants des rues.

Le père, le fils et le saint Torum, 1997.

Documentaire de Mark Soosar. Prix Nanook du Bilan du film ethnographique. Non diffusé en France.

Le conflit entre un chamane khante et son fils qui travaille dans l'industrie.

Shaman, 1993.

Documentaire d'Elisabeth Wennberg, 55 min. Danemark, télévision danoise.

Les Nganassanes et leur chamane.

Spiritualité : chamane à Moscou, 1996.

Reportage de Thomas Johnson, 12 min. Prod. Canal Plus et Capa production, diff. Canal Plus, 1996. 
Albert, un ancien rockeur d'origine sibérienne, accomplit un rite dans la taïga, et donne un concert de musique techno dans une boîte de nuit moscovite. Il pratique le chant de gorge.

Taïga, terre des chamans, 1996.

Documentaire de Catherine et Marc Garanger, 52 min. Prod. France 3, ADR, Paris, diff. France 3, 1997.

Enquête chez les Évenks de Iakoutie.

Trois chamanes, 1994.

Documentaire de Mark Soosar. Non diffusé en France.

Comparaison entre Vigala Sass, personnage légendaire estonien, un chef séquoia, et un chamane khante.

Les Tsaatan, ceux qui chevauchent les rennes, 1995.

Documentaire de Jacques Malaterre, 26 min. Prod.-diff. Canal Plus, 1995 et La Cinquième, 1998.

La vie de Bat, chef des Tsaatanes et fils de chamane, dont le renne sacré, désigné par son père, vient à mourir.

Le voyage de Charlie, 2001.

Documentaire de Stéphane Bégoin, 52 min. Prod. La Compagnie des Taxis-Brousse, Paris, L'Amour en l'an 2000, Québec, diff. Arte, 2002.

Charlie, un travailleur social inuit, voyant son peuple perdre son identité va suivre l'enseignement d'un chamane shipibo, Guillermo. Des anthropologues français ont servi d'intermédiaires.

Xingu, le corps et les esprits, 1996.

Documentaire de Marie Correa, 54 min. Prod. Les Films du village, Paris, diff.

La Cinquième, 1997. En Amazonie, dans le Xingu, une mission médicale se partage les tâches avec un chamane.

\section{NOTES}

1. On trouvera dans la filmographie un bref synopsis pour la plupart des films cités.

2. Il arrive cependant depuis une période récente que les indigènes, pour des raisons stratégiques, s'appliquent le nom de «chamane», venu par l'anglais ou l'espagnol. Simultanément, ils endossent aussi les traits de la figure occidentale du chamane, ce qui ne fait que faciliter la tâche des fabricants de films.

3. Interview de Martine Journet et Gérard Nougarol sur le site http://mapage.noos.fr/chamati/ assoc.html

4. Interview de Martine Journet et Gérard Nougarol sur le site http://mapage.noos.fr/chamati/ assoc.html

5. Ici fait surface un principe essentiel de la vision occidentale du chamane, contre lequel les indigènes ne peuvent opposer de résistance. Il est notable que, d'après les chamanes touvas par exemple, la compréhension des chants chamaniques par l'assistance est indispensable.

6. Profondeur de champ: profondeur de la zone où les objets apparaissent nets. La réduire permet d'isoler un élément en mettant ce qui est derrière lui dans le flou.

7. Très gros plan sur un détail, souvent de brève durée.

8. Annick Rivoire, "Vue de l'esprit yanomami ", Libération du 28 août 2003, pp. 28-29. Comme l'indique subtilement le titre de l'article, on va à cette exposition pour voir l'esprit (chamanique) que les Yanomamis ont à l'esprit.

9. Reportage du journal télévisé du 22 mai 2003 à 20 h05. 
10. On retrouve ce parallèle dans un texte important du poète contemporain Denis Roche qui, après avoir cité Eliade, écrit : «Les mêmes phénomènes qui présidaient autrefois aux révélations du sorcier sibérien président aujourd'hui aux inventions du poète moderne " (ROCHE 1995, p. 9).

11. «Le chamane entre dans un état de conscience modifié qui lui permet de communiquer avec les forces cachées de la nature " (site officiel du film Blueberry : http://www.blueberry-lefilm.com/\#) 12. Interview de Stéphane Bégoin par Sabine Lange publiée sur le site http:// www.informactionfilms.com/fr/accueil/index.html

13. "On appelle couramment in le son dont on peut vérifier le synchronisme avec l'image, le son qui a un double visuel : le plus souvent lèvres qui articulent, mais aussi porte qui claque, mains qui applaudissent, etc.; et off le son dont la source n'est pas présente à l'image » (villain 2001, p. 90).

14. Ce film a reçu le prix Nanook du Bilan du film ethnographique. Cependant, on peut s'interroger sur le caractère ethnographique de son travail, comme le fait Éva Toulouze dans France-Estonie, 11, 1998: "Mark Soosaar, plus qu'un chercheur résolu à livrer des matériaux importants en soi et utilisables y compris par d'autres (ce qui est l'objectif de l'ethnologue), est un fabricant de contes moraux. "

15. Le son postsynchronisé remplace ou s'ajoute au son direct qui a été enregistré en même temps que l'image.

16. L'insistance du fabricant de film sur la maîtrise que les chamanes ont de la botanique et spécialement de la botanique psychodysleptique n'est pas seulement l'expression d'un anticonformisme convenu opposant à la sévérité des lois occidentales un relativisme généreux. L'utilisation de psychotropes est donnée souvent comme explication mécanique de la transe. Si le chamane se doit d'être un bon herboriste, c'est parce que ses visions, et donc les représentations de sa société sur les esprits, trouvent dans les plantes leur cause matérielle. L'Occidental peut dès lors espérer pénétrer lui aussi dans le monde des esprits, grâce à une double initiation simultanée - à la drogue locale et aux représentations indigènes. On en verra plus loin quelques exemples. Wittgenstein a montré les difficultés que soulève l'application d'une explication mécaniste à un phénomène culturel. C'est isoler un fait signifiant du champ général des significations pour lui donner son sens uniquement dans ses effets. Si le sens d'une pratique culturelle est tout en entier contenu dans l'expérience kinesthésique qui l'accompagne (émotion esthétique, vision mystique), alors on pourrait imaginer de prendre une seringue qui nous ferait le même effet que la contemplation d'un tableau ou l'audition d'un cantique (wittgenstein 1992, pp. 66-67). On n'est pourtant pas tenté de le faire. L'usage des psychotropes donne naturellement facilement à croire que le sens et la fonction des pratiques chamaniques sont contenus dans une expérience sensible privée de nature exceptionnelle.

17. Pour les caméras vidéo, teinte générale de l'image donnée par une référence de blanc.

18. Dans le film, il s'agit d'inhalation et non de boisson. Interview de Stéphane Bégoin par Sabine Lange publiée sur le site http://www.informactionfilms.com/fr/accueil/index.html

19. Interview des réalisateurs sur le site $h t t p: / / m a p a g e . n o o s . f r / c h a m a t i / a s s o c . h t m l$

20. C'est l'expression choisie pour traduire les mots de la jeune fille : «Takoj vostorg!»

21. La Fondation Cartier a également acheté au producteur de ce fameux groupe sibérien des droits de diffusion pour utiliser certains chants comme fond sonore d'images sur les Yanomamis.

22. Site de Stéphane Bégoin : http://www.stephanebegoin.com

23. Site officiel du film : http://deathofashaman.com/

24. Même si cette opinion est authentique, elle est peu représentative de la situation des peuples indigènes de Sibérie, dont beaucoup de jeunes gens, au chômage, s'engagent comme soldats professionnels en Tchétchénie, et lorsqu'ils sont pris subissent des représailles particulièrement cruelles de la part des combattants tchétchènes qui les considèrent comme des mercenaires, et épargnent en revanche les appelés du contingent. Le parti pris du fabricant de film, cherchant à faire bénéficier les Évenks de l'émotion suscitée par le sort des Tchétchènes, est évident. 
25. Pod pressom gosudarstva. Il faut signaler que, au contraire, au début des années 1990 , l'hypothèse avait été sérieusement examinée de faire du chamanisme la religion officielle de la république de Iakoutie, où le film était tourné en 1996.

26. http://www.blueberry-lefilm.com/\#

La mythologie occidentale sur le sujet se distingue de celle des sociétés traditionnelles, notamment par la puissance incomparable de son efficacité symbolique. Jan Kounen, après l'expérience rebelle de Doberman, a voulu aborder la question de «[sa propre] place dans l'univers» et s'est mis en quête de ce qu'il nomme "d'autres dimensions». À cette fin, un support idéologique, ou « filtre ", lui était nécessaire, et ses lectures l'orientèrent vers le système de pensée qui lui semblait le moins contraignant et le plus individualiste dans ses méthodes, le chamanisme. De ses lectures, il retira une représentation des chamanes que nous avons déjà rencontrée, faite de pédagogie et de soin psychothérapique : «Leur rôle : guider les âmes dans leur quête personnelle. » Conséquemment, il partit pour le Pérou à la recherche d'un " maîtrechamane "; le chamane qui accepta de l'adopter ne fut autre que Guillermo (ou Kestembetsa en langue shipibo), le héros amazonien du Voyage de Charlie. Très satisfait de l'initiation, et en particulier de la découverte des effets de la fameuse plante psychotrope ayahuasca, Kounen avait déjà ainsi actualisé pour son propre compte le mythe occidental, grâce à l'aide complaisante d'un entrepreneur d'identité habitué à rendre ces services au visiteur. Par la suite, désireux de donner au mythe une diffusion internationale, Kounen réalisa le film de fiction Blueberry, où l'on voit un Blanc initié, comme lui, par un chamane indien. Là où l'efficacité symbolique du mythe occidental apparaît dans toute l'ambiguïté de sa violence bien intentionnée, c'est dans le fait que la personne choisie pour jouer le rôle du chamane arizonien fut Guillermo lui-même. Ce fait est peu connu, car, pour des raisons obscures, cette participation fut ensuite largement occultée, et Guillermo ne figure pas dans le casting officiel, bien qu'il apparaisse à l'écran. Kounen répara, du reste, cette omission en parachevant la transformation de Guillermo en "guide spirituel ", l'invitant à Paris à plusieurs reprises pour tenir des conférences sur le chamanisme et consacrant à son maître un documentaire, Other worlds, qui, secondé de puissants effets spéciaux, se donne pour but de faire découvrir « une psychanalyse ancestrale, une psychothérapie de l'être ayant 4000 ans d'expérimentation » (sortie le 30 juin 2004).

[Les citations sont tirées du site http://otherworlds.jankounen.com/\#]

27. Voyage en Sibérie, dans Charles de Saint-Julien, Voyage pittoresque en Russie, Quatrième partie, chapitres II et III, 1853 (extraits recueillis dans de Grève 1990, pp. 664, 667-668).

28. Sur le site de la Fondation Cartier: http://www.fondation.cartier.fr/fra/expo/yanomami/ depardon.htm

\section{RÉSUMÉS}

Le chamanisme, notion de vulgarisation récente qui tend à recouvrir celle de sorcellerie, est l'objet dans nos sociétés d'une fascination croissante. Le chamane est devenu le héros d'un véritable mythe dont il est intéressant de recueillir les traits caractéristiques. En particulier, les productions audiovisuelles sont autant de variantes du mythe qui, en plus de le reformuler, lui donnent une confirmation objectivante en vertu de la transparence présumée de l'image. Mettant en doute un tel postulat, cet article s'attache à analyser dans plusieurs productions diffusées en France le travail efficace d'un mythe savant. 
Shamanism, a recently popular idea that tends to overlap with that of sorcery, is an object of growing interest in our society. The shaman has become the hero of an actual myth, whose characteristic traits the article is interested in assembling. In particular, audiovisual productions present variants of this myth which, in addition to reformulating the myth, give to it an objectifying confirmation by virtue of the presumed transparency of the image. Calling this postulate into question, the article seeks to analyse the effective employment of a scholarly myth in several productions broadcast in France.

\section{INDEX}

Thèmes : chamanisme

Keywords : audiovisual productions, cinema, image, representation of the other, shaman

Mots-clés : chamane, cinéma, image, productions audiovisuelles, représentation de l'autre

Index géographique : Sibérie

\section{AUTEUR}

\section{CHARLES STÉPANOFF}

Ancien élève de l'ENS, docteur de l'EPHE en Anthropologie religieuse (2007), maître de conférences à l'EPHE (2008). Auteur d'un film, Touva, république des chamanes 